\title{
A minimal non-solvable group of homeomorphisms
}

\author{
Collin Bleak
}

\begin{abstract}
Let $\mathrm{PL}_{o}(I)$ represent the group of orientation-preserving piecewise-linear homeomorphisms of the unit interval which admit finitely many breaks in slope, under the operation of composition. We find a non-solvable group $W$ and show that $W$ embeds in every non-solvable subgroup of $\mathrm{PL}_{O}(I)$. We find mild conditions under which other non-solvable subgroups $B$, $(\zeta \mathbb{Z} 乙)^{\infty},(\mathbb{Z} 乙)^{\infty}$, and $\left.{ }^{\infty}(\zeta \mathbb{Z})\right)$ embed in subgroups of $\operatorname{PL}_{o}(I)$. We show that all solvable subgroups of $\mathrm{PL}_{o}(I)$ embed in all non-solvable subgroups of $\mathrm{PL}_{o}(I)$. These results continue to apply if we replace $\mathrm{PL}_{o}(I)$ by any generalized Thompson group $F_{n}$.
\end{abstract}

Mathematics Subject Classification (2000). 20F62,37E05, 20F38, 20E07, 20E22.

Keywords. PL homeomorphisms, group actions, unit interval, non-solvable groups, Thompson's group $F$.

\section{Introduction}

Let the symbol $\mathrm{PL}_{o}(I)$ represent the group of orientation-preserving piecewise-linear homeomorphisms of the unit interval which admit finitely many breaks in slope, under the operation of composition. We show that there is a non-solvable group $W$ so that $W$ embeds in every non-solvable subgroup of $\mathrm{PL}_{o}(I)$. We show that every solvable subgroup of $\mathrm{PL}_{o}(I)$ embeds in every non-solvable subgroup of $\mathrm{PL}_{o}(I)$ (see [3] and [2] for a geometric and two algebraic classifications of the solvable subgroups of $\left.\mathrm{PL}_{o}(I)\right)$. We show that all virtually solvable subgroups of $\mathrm{PL}_{o}(I)$ are in fact solvable. Finally, if $H \leq \mathrm{PL}_{o}(I)$, we find various mild conditions on the action of $H$ on the unit interval which imply the existence in $H$ of embedded copies of various of the non-solvable

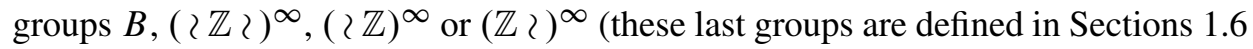
and 1.8).

We note that $\mathrm{PL}_{o}(I)$ has received attention from various researchers lately, primarily because it is "a source of groups with interesting properties in which calculations are practical" (quoting Brin and Squier in [8]). For example, $\mathrm{PL}_{o}(I)$ contains copies of each of the generalized Thompson groups $F_{n}$, which have themselves been a focus of current research. (The groups $F_{n}$ were introduced by Brown in [9], where they were denoted $F_{n, \infty}$. R. Thompson's group $F$ corresponds to $F_{2}$ in this context. The 
family of groups $F_{n}$ are further studied by Stein in [11], by Brin and Guzmán in [6] and by Burillo, Cleary, and Stein in [10].)

All of our stated results for subgroups of $\operatorname{PL}_{o}(I)$ hold if we replace the group $\mathrm{PL}_{o}(I)$ in each of the statements with any particular group $F_{n}$.

This paper is a logical continuation of the investigations began in [3] and continued in [2], and it indirectly uses some machinery developed in [5]. While we will only need a small part of the theory developed in [2], we will need almost all of the definitions, theory, and techniques developed in [3]. Instead of simply restating the whole of [3], we will assume that the reader is familiar with that paper, although we will restate relevant definitions here.

Our purely algebraic results are stated in Section 1.1, while our geometric results are stated in Section 1.2, after we give the required definitions. We note in passing that the proof of our main algebraic result depends in large part on our geometric results.

The author would like to thank Matt Brin and Binghamton University for their support during the research leading up to this paper. The author would also like to thank his referees, who gave many detailed and helpful comments; they significantly helped the author to improve the exposition of this article.

Some of the results here are contained in the author's dissertation written at Binghamton University.

1.1. Our main algebraic result. In the context of $F$, there have been two prime candidates for minimal non-solvable subgroups; the groups denoted by $\infty(২ \mathbb{Z})$ and $(\mathbb{Z} \imath)^{\infty}$ in [5]. In particular, Sapir had asked the question of whether every nonsolvable subgroup of $F$ contained a copy of $(\mathbb{Z} 2)^{\infty}$. Brin in [5] answers this negatively by showing that both $(\mathbb{Z} \imath)^{\infty}$ and ${ }^{\infty}(\imath \mathbb{Z})$ occur as embedded subgroups of $F$, and that neither of these two groups contains the other as an embedded subgroup. Brin then asks (Question 1 of his text) whether one of these two groups has to occur as an embedded subgroup in any non-solvable subgroup of $\operatorname{PL}_{o}(I)$.

Our chief result is the following.

Theorem 1.1. Let $H$ be a subgroup of $\mathrm{PL}_{o}(I) . H$ is non-solvable if and only if $W$ embeds in $H$.

Since both ${ }^{\infty}(\succ \mathbb{Z})$ and $(\mathbb{Z} \succ)^{\infty}$ fails to embed in the other it is an immediate corollary of our Theorem 1.1 that neither will embed into $W$. Thus, we answer Brin's question negatively.

There is also a finitely generated group $B$, denoted $G_{1}$ in Section 5 of [5] (where Brin introduces the group), which contains both ${ }^{\infty}(\succ \mathbb{Z})$ and $(\mathbb{Z} \imath)^{\infty}$. We state the following conjecture, which, in the light of our Theorem 1.4, is really a just a promotion of Question 1 from [3].

Conjecture. Any finitely generated non-solvable subgroup of $\mathrm{PL}_{o}(I)$ contains an embedded copy of the group $B$. 
1.2. Key algebraic definitions. We now work to define $W$, and then we state some immediate corollaries of Theorem 1.1.

We must first recall the definition of a standard restricted wreath product of groups.

Let $C$ and $T$ be groups. Let $M=\bigoplus_{t \in T} C$ represent the direct sum of copies of $C$ indexed by the elements of $T$. We will denote the group $M \rtimes T$ (where the action of $T$ on $M$ is by right multiplication on the indices) by the symbol $C<T$. The group $C \geqslant T$ is the standard restricted wreath product of $C$ and $T$. Following standard convention, we will refer to $C$ as the "bottom group" of $C<T$, we will refer to $M$ as the "base group" of $C<T$, and finally we will refer to $T$ as the "top group" of $C<T$. As we will not have a need to explicitly discuss other types of wreath products in this paper, we will use the phrase "wreath product" to mean the "standard restricted wreath product" in the remainder. Note that we can think of $C, M$, and $T$ as subgroups of $C>T$ in fairly obvious manners (a little care is required when choosing the realization of the group $C$ in $C>T$, as many candidate copies of $C$ are available).

If $C$ is a subgroup of $\mathrm{PL}_{o}(I)$, then there is a straightforward geometric construction that realizes the group $C \imath \mathbb{Z}$ in $\mathrm{PL}_{o}(I)$. We will give a concrete demonstration of this construction below in Section 1.8. The construction is so basic in $\mathrm{PL}_{o}(I)$ that it motivates the definition of the following family of groups (the groups $\left(W_{i}\right)_{i=0}^{\infty}$ mentioned above), which will play a central role in all that follows.

Define

$$
W_{0}=\{1\} \quad \text { and } \quad W_{n}=W_{n-1}<\mathbb{Z} \text { for } n>0 \text { with } n \in \mathbb{N} .
$$

Note here that we use $\mathbb{N}=\{0,1,2, \ldots\}$.

We are now ready to define the group $W$ :

$$
W=\bigoplus_{n \in \mathbb{N}} W_{n}
$$

1.3. Corollaries of the chief algebraic result. It is a consequence of the main results of [2] that each solvable subgroup $H$ of $\operatorname{PL}_{o}(I)$ admits an $n \in \mathbb{N}$ so that $H$ embeds in $W_{n}$. However, each $W_{n}$ embeds in $W$. Therefore, Theorem 1.1 has the following consequence.

Corollary 1.2. Every solvable subgroup of $\mathrm{PL}_{o}(I)$ embeds in every non-solvable subgroup of $\mathrm{PL}_{o}(I)$.

Note that the above statement is also true if we replace $\mathrm{PL}_{o}(I)$ by $F$ in both locations.

Since $W$ is not virtually solvable, we see that the non-solvable subgroups of $\mathrm{PL}_{o}(I)$ are not virtually solvable; in particular we have the following second corollary.

Corollary 1.3. Suppose that $H$ is a subgroup of $\mathrm{PL}_{o}(I)$. If $H$ is virtually solvable, then $H$ is solvable. 
As mentioned above, the papers [3] and [2] provide both geometric and algebraic classifications of the solvable subgroups of $\mathrm{PL}_{o}(I)$. Therefore, the last corollary, together with the cited research, is sufficient to classify the virtually solvable subgroups of $\mathrm{PL}_{o}(I)$.

1.4. General geometric definitions. In order to state our more geometric results in a meaningful fashion, we must give some definitions.

Throughout this section and the next, we fix a model group $G \leq \mathrm{PL}_{o}(I)$.

Define Supp $G$, the support of $G$, to be the set $\{x \in I \mid x g \neq x$ for some $g \in G\}$. The set Supp $G$ is an open subset of $(0,1)$, and can therefore be written as a disjoint union of a countable (possibly finite) collection of open intervals in $(0,1)$. If $g \in G$ then we will similarly refer to $\operatorname{Supp}\langle g\rangle$ as the support of $g$. We note in passing that if $g, h \in G$ and the support of $g$ and the support of $h$ are disjoint, then $h$ and $g$ commute. We call any interval component of Supp $G$ an orbital of $G$. If $g \in G$, and $A$ is an orbital of $\langle g\rangle$, then we say $A$ is an orbital of $g$ or an element-orbital of $G$.

We also note that if $g \in G$ and $A$ is an orbital of $g$ then either $g$ moves all points in $A$ to the right or $g$ moves all points in $A$ to the left. If $g$ moves all points in $A$ to the right and $x \in A$ then we will refer to the interval $[x, x g$ ) as a fundamental domain of $g$ in $A$. (Note: in this paper, all group actions will be right actions, and also that we will compose elements from the left to the right). If $g$ moves all points in $A$ to the left, then we will similarly refer to the interval $\left(x, x g^{-1}\right]$ as a fundamental domain of $g$ in $A$. We will occasionally not mention the orbital $A$ if the context will allow us to do this without confusion.

Note that a fundamental domain of an element $g \in G$ in one of its orbitals $A$ is a maximal subinterval of $A$ that is entirely mapped off of itself by the action of $g$.

If $A$ is an element-orbital of $G$, there will be infinitely many elements in $G$ with orbital $A$, so that we often explicitly associate an element with an element-orbital. We call an ordered pair $(A, g)$ a signed orbital of $G$ if $g$ is an element of $G$ with orbital $A$. In this case, we refer to $A$ as the orbital of $(A, g)$ and $g$ as the signature of $(A, g)$. We will often work with sets of signed orbitals. If $X$ is a set of signed orbitals, then we will sometimes form sets $O_{X}$ and $S_{X}$, where $O_{X}=\{A \subset I \mid$ $(A, g) \in X$ for some $g \in G\}$ and $S_{X}=\{g \in G \mid(A, g) \in X$ for some $A \subset I\}$. We will refer to the set $O_{X}$ as the orbitals of $X$ and to the set $S_{X}$ as the signatures of $X$. We may also consider the group $G_{X}=\left\langle S_{X}\right\rangle$, the group generated by the set of signatures of $X$.

1.5. Transition chains. Suppose that $\mathcal{C}=\left\{\left(A_{p}, g_{p}\right) \mid p \in d\right\}$ is a set of signed orbitals indexed by a set $d \subset I$ and

$$
A_{e}=\bigcup_{p \in \mathcal{l}} A_{p} .
$$

We call $\mathcal{C}$ a transition chain if $\mathcal{C}$ satisfies the following conditions: 
(1) For all $x, y \in A_{e}$, with $x<y$, the interval $[x, y] \subset A_{\complement}$.

(2) For each $p \in \mathcal{d}$, we have

(a) $p \in A_{p}$, and

(b) if $q \in d$ and $p \in A_{q}$, then $p=q$.

In this case, we refer to the cardinality of $d$ as the length of $\mathcal{C}$.

The index set $d$ plays another role. As any two points in the index set are comparable using the total order $\leq$ on $(0,1)$, we have an induced total order on the set of signed orbitals of $\mathcal{C}$. For finite transition chains, we will often emphasize this total order by implicitly passing to a new index set of natural numbers, so that we can refer to the "first" orbital in the transition chain (or simply, in the chain), or the "second", or the "last." In fact, we generally only explicitly define the initial index set when we need to use the points of that index set in some specific way, having to do with the action of $G_{e}$ on $[0,1]$. These practices should cause no confusion to the reader.

The point of a transition chain is that $A e$ is an open interval in $(0,1)$ on which the signatures of $\mathcal{C}$ may act non-trivially. Further, it is immediate that by carefully choosing which signatures to act with (including using inverses of signatures), and in some specific order, we can find an element in $G e$ which moves any particular point in $A_{e}$ as far to the left or right in $A_{e}$ as we wish.

In the case of short transition chains, we will have the second condition fairly easily from direct considerations. For instance, here is an alternative specific definition of a transition chain of length two. Suppose that $\mathcal{C}=\{(A, g),(B, h)\}$ is a set of signed orbitals of $G$, where $A=\left(a_{l}, a_{r}\right), B=\left(b_{l}, b_{r}\right)$ and $a_{l}<b_{l}<a_{r}<b_{r}$. In this case we call $C$ a transition chain of length two for $G$.

We now release the group $G$. We will use the language developed above freely with other subgroups of $\mathrm{PL}_{o}(I)$, expecting that this will lead to no confusion.

1.6. A graphical shorthand. In general, we will often work with multiple elements in $\operatorname{PL}_{o}(I)$ at one time. In these cases it is extremely useful to be able to visualize how the supports of the elements are overlapping, and in what directions points in the interval move under the action of these elements. In this short section we indicate a diagram "shorthand" that we will often use in order to demonstrate ideas in this paper.

The essential concept is that we will replace a graph of a function $f:[0,1] \rightarrow[0,1]$ by a rough version; we essentially sketch a version of the graph of $f-$ id, where id is the identity function. In our diagram, we will not sketch the horizontal regions of $f$-id corresponding to value 0 , and we will forget that $f$ is (generally) piecewise linear. We will, however, be very careful to keep track of the ends of the orbitals of $f$, not allowing them to move left or right in our sketch.

Below is a graph of an example pair of functions, and then, allowing ourselves to further offset results vertically, we will have a labeled diagram of the same pair. 


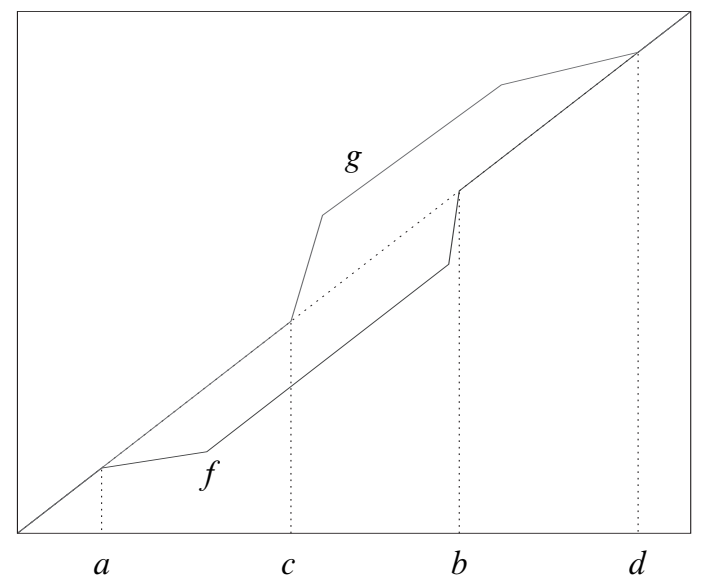

Below we present an example diagram of the transition chain of length two generated by the functions graphed above.

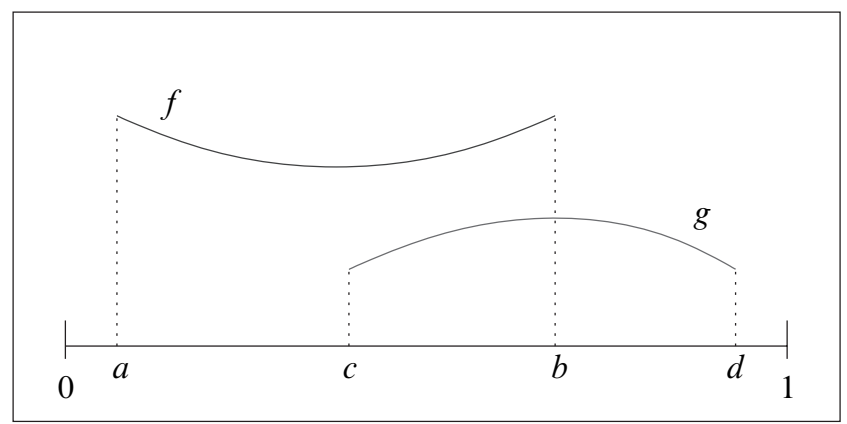

1.7. The group $B$, and our chief geometric result. With transition chains defined, we only need a definition of the group $B$ in order to state our first geometric result. Recall that the group $B$ was introduced in a general form in [5] under the notation $G_{1}$ in Section 5 of that paper. Our realization will be much more concrete, but as in [5], it will still be realized in Thompson's group $F$ (the subgroup of $\mathrm{PL}_{o}(I)$ consisting of elements whose breaks in slope occur only in the dyadic rationals, and which have all slope values powers of 2).

Define $\alpha \in \mathrm{PL}_{o}(I)$ to be the element so that given any $x \in I$, we have

$$
x \alpha= \begin{cases}\frac{1}{4} x, & 0 \leq x<\frac{1}{4} \\ x-\frac{3}{16}, & \frac{1}{4} \leq x<\frac{7}{16} \\ 4 x-\frac{3}{2}, & \frac{7}{16} \leq x<\frac{9}{16} \\ x+\frac{3}{16}, & \frac{9}{16} \leq x<\frac{3}{4} \\ \frac{1}{4} x+\frac{3}{4}, & \frac{3}{4} \leq x \leq 1\end{cases}
$$


and define $\beta_{0} \in \mathrm{PL}_{o}(I)$ to be the element so that given any $x \in I$, we have

$$
x \beta_{0}= \begin{cases}x, & 0 \leq x<\frac{7}{16} \\ 2 x-\frac{7}{16}, & \frac{7}{16} \leq x<\frac{15}{32} \\ x+\frac{1}{32}, & \frac{15}{32} \leq x<\frac{1}{2} \\ \frac{1}{2} x+\frac{9}{32}, & \frac{1}{2} \leq x<\frac{9}{16} \\ x, & \frac{9}{16} \leq x \leq 1\end{cases}
$$

The graphs of these elements (superimposed) are given below.

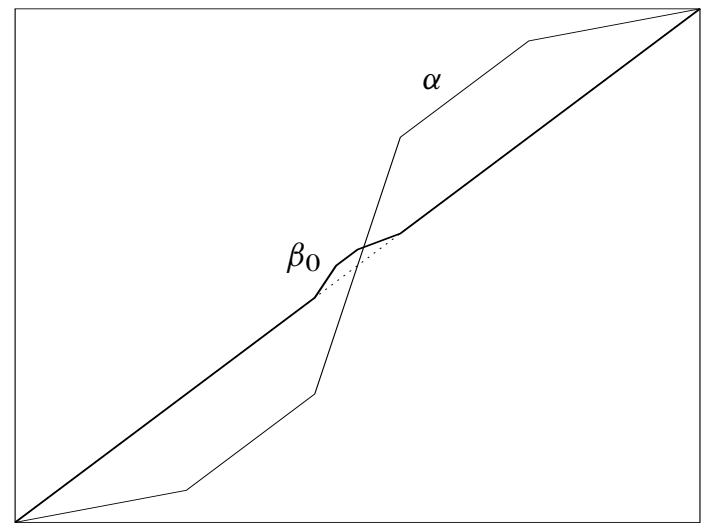

We define

$$
B=\left\langle\alpha, \beta_{0}\right\rangle .
$$

We show, via a strengthening of an argument in [3], the following result.

Theorem 1.4. If $H \leq \mathrm{PL}_{o}(I)$ admits a transition chain of length two, then $B$ embeds in $H$.

It is extremely restrictive to only consider subgroups of $\mathrm{PL}_{o}(I)$ which do not admit transition chains of length two. In particular, $B$ embeds in "most" naturally occurring subgroups of $\mathrm{PL}_{o}(I)$.

1.8. The structure of the group $\boldsymbol{B}$. We would like to discuss $B$ further, and extend our chief geometric result, but we need to establish a few more conventions before we proceed. Let $x \in I$ and $g, h \in \mathrm{PL}_{o}(I)$, and recall that all group actions will be written as right actions (so that $x g$ is the point $x$ goes to when acted upon by $g$ ) and that compositions will occur from the left to the right. We will represent the conjugate of $g$ by $h$ by the expression $g^{h}$, which will mean $h^{-1} g h$, and the commutator of $g$ and $h$ by the expression $[g, h]$, which will mean $g^{-1} h^{-1} g h$. 
Let us now discuss the group $B$. One perspective on the group $B$ is that the element $\alpha$ acts as a "Growing conjugator", conjugating $\beta_{0}$ to new elements $\left\{\beta_{i}\right\}_{i \in \mathbb{Z}}$ (which, for positive index $i$, have larger supporting sets). We demonstrate this behavior, and explore the structure of the groups generated by sub-collections of the $\beta_{i}$.

Define $\beta_{k}=\beta_{0}^{\alpha^{k}}$ for each integer $k$. In particular, $\beta_{1}$ is given by the rule

$$
x \beta_{1}=x \alpha^{-1} \beta_{0} \alpha= \begin{cases}x, & 0 \leq x<\frac{1}{4}, \\ 2 x-\frac{1}{4}, & \frac{1}{4} \leq x<\frac{3}{8} \\ x+\frac{1}{8}, & \frac{3}{8} \leq x<\frac{1}{2} \\ \frac{1}{2} x+\frac{3}{8}, & \frac{1}{2} \leq x<\frac{3}{4} \\ x, & \frac{3}{4} \leq x \leq 1 .\end{cases}
$$

Observe that the support of $\beta_{0}$ is $\left(\frac{7}{16}, \frac{9}{16}\right)$, and since $\frac{7}{16} \beta_{1}=\frac{9}{16}$, the support of $\beta_{0}$ is contained in a single fundamental domain of $\beta_{1}$. In particular, given any $i \in \mathbb{Z}$, we see that the support of $\beta_{i-1}$ is contained in a single fundamental domain of the support of $\beta_{i}$, since these two elements are conjugates of $\beta_{0}$ and $\beta_{1}$. Therefore, given an $i \in \mathbb{Z}$, any two conjugates of $\beta_{i}$ by distinct powers of $\beta_{i+1}$ will have disjoint support, so that these two conjugates will commute. In particular, the group $\left\langle\beta_{i}, \beta_{i+1}\right\rangle$ is isomorphic with $\mathbb{Z} \imath \mathbb{Z}$. More generally, given an $n \in \mathbb{N}$, we see that any collection of $n$ distinct $\beta_{i}$ will generate a group isomorphic with $W_{n}$.

Let $\mathcal{W}=\left\{\beta_{i}\right\}_{i \in \mathbb{Z}}$ throughout the paper.

In passing, let us point out that if $G$ is a subgroup of $\operatorname{PL}_{o}(I)$, then by considering $G$ to be a subgroup of Homeo $(\mathbb{R})$ (extend the elements of $G$ by using the identity function outside of the unit interval), we can conjugate $G$ (by a conjugator in Homeo $(\mathbb{R})$ ) to $\widehat{G}$, a piecewise linear copy of $G$ whose support is in $\left(\frac{7}{16}, \frac{9}{16}\right)$. By the discussion above, we see that the group $\left\langle\widehat{G}, \beta_{0}\right\rangle$ is therefore isomorphic to $G \geq \mathbb{Z}$. This is a concrete version of the construction mentioned earlier in the introduction.

We can think of $B$ as an HNN extension of the group generated by the full collection $\mathcal{W}$ of the $\beta_{i}$, where $\alpha$ plays the role of the stable letter, with the rule that $\beta_{i}^{\alpha}=\beta_{i+1}$ for each integer $i$. This then gives us a criterion for detecting when a two-generator (say $\omega_{0}$ and $\gamma$ ) subgroup of $\mathrm{PL}_{o}(I)$ is isomorphic to $B$. For each integer $i$, define $\omega_{i}=\omega_{0}^{\gamma^{i}}$. The group $\left\langle\omega_{0}, \gamma\right\rangle$ will be isomorphic to $B$ if the set map $\Upsilon:\left\{\omega_{i}\right\}_{i \in \mathbb{Z}} \rightarrow\left\{\beta_{i}\right\}_{i \in \mathbb{Z}}$, defined by the rule $\omega_{i} \mapsto \beta_{i}$ for all integers $i$, is well defined (as we are considering elements of $\mathrm{PL}_{o}(I)$, this last boils down to $\gamma$ and $\omega_{0}$ not commuting), and extends to a group isomorphism $\left\langle\left\{\omega_{i}\right\}_{i \in \mathbb{Z}}\right\rangle \cong\langle\mathcal{W}\rangle$. Note that the last isomorphism is easy to detect geometrically via the tools developed in [5]; in our case, if the closure of the support of the non-trivial $\omega_{0}$ is fully contained in a set $X_{0}$, and $X_{0} \omega_{1} \cap X_{0}=\emptyset$, then $\left\langle\left\{\omega_{i}\right\}_{i \in \mathbb{Z}}\right\rangle \cong\langle\mathcal{W}\rangle$. 
1.9. Towers, and further results. Our last set of geometric results is mostly relevant in the situation where a subgroup of $\mathrm{PL}_{o}(I)$ fails to admit transition chains of length two. In this restrictive case, we use other sets of signed orbitals to understand our group structures.

It is commonly known that $\mathrm{PL}_{o}(I)$ is totally ordered, and that the set of open intervals in $I$ is a poset under inclusion. Therefore we can define a partial ordering on the set of signed orbitals of $\mathrm{PL}_{o}(I)$. If $(A, f)$ and $(B, g)$ are signed orbitals of $\mathrm{PL}_{o}(I)$, then we will say that $(A, f)<(B, g)$ if either (1) $A \subsetneq B$ or (2) $A=B$ but $f<g$. We will use this partial ordering to help form the following definition.

Given a subgroup $G$ of $\mathrm{PL}_{o}(I)$, we say that a set $\mathcal{T}$ of signed orbitals of $G$ is a tower associated with $G$ if $\mathcal{T}$ satisfies two properties.

(1) $\mathcal{T}$ is a chain in the poset of signed orbitals of $\operatorname{PL}_{o}(I)$.

(2) If $A \subset I$ and $(A, g)$ and $(A, h)$ are in $\mathcal{T}$, then $g=h$.

Note in passing that the second condition above assures us that orbitals in a tower get larger as we move to larger elements in the tower.

We define the height of a tower to be its cardinality. We define the depth of a subgroup $G$ of $\mathrm{PL}_{o}(I)$ to be the supremum of the set of cardinalities of towers associated with $G$.

Consider the graph of three functions (superimposed) below.

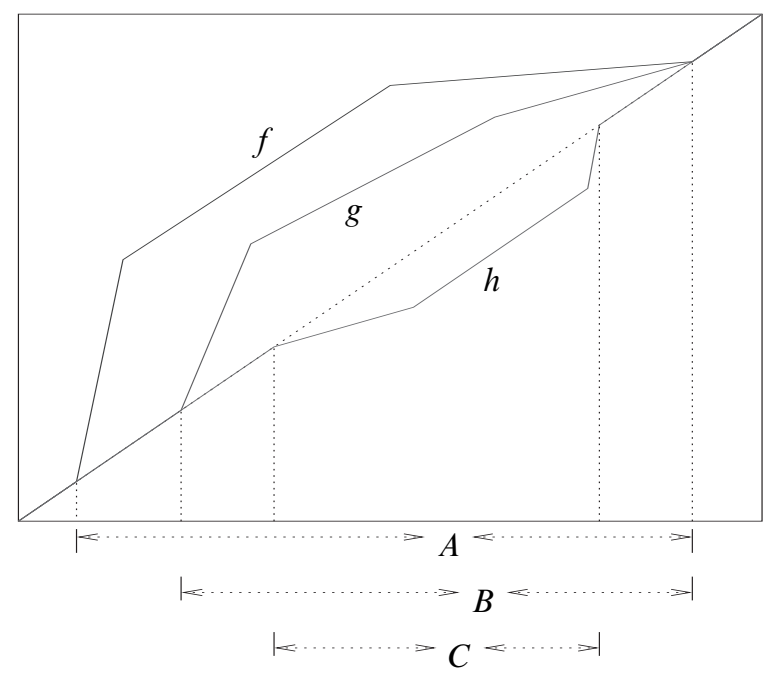

With these three elements of $\mathrm{PL}_{o}(I)$, we can create the tower

$$
T=\{(A, f),(B, g),(C, h)\} .
$$

In this case, note that the signed orbitals of the tower $T$ are listed in reverse of their order in the poset of signed orbitals of $\mathrm{PL}_{o}(I)$. 
If $G$ is a subgroup of $\mathrm{PL}_{o}(I)$ which does not admit infinite towers, then we also assign depth to any signed orbital $(A, g)$ of $G$, as the maximum cardinality of the towers which contain $(A, g)$ as their least element. (We leave it to the reader to check that this is well defined, that is, if $(A, g)$ is the smallest element in infinitely many finite towers for $G$, with arbitrarily large height, then $G$ admits an infinite tower with least element $(A, g))$. The following is a variation of the main result of [3].

Theorem 1.5. Suppose that $H$ is a subgroup of $\mathrm{PL}_{o}(I) . H$ is non-solvable if and only if $H$ admits towers of arbitrary finite height.

This last result assures us that if $H \leq \mathrm{PL}_{o}(I)$ admits an infinite tower, then $H$ will be non-solvable. We now focus on the sorts of subgroups we can find in such a group $H$, depending on the types of transition chains and towers we can find associated with $H$.

If a tower $T$ admits an order-preserving injection $t: \mathbb{N} \rightarrow T$, then we will say that the tower is tall. If a tower $T$ admits an order reversing injection $d: \mathbb{N} \rightarrow T$, then we will say that a tower is deep. If a tower $T$ admits an order-preserving injection $b: \mathbb{Z} \rightarrow T$ then we will say $T$ is bi-infinite.

We are about ready to state our last set of results; we first need concrete realizations of the groups ${ }^{\infty}(\zeta \mathbb{Z})$ and $(\mathbb{Z} \imath)^{\infty}$, as well as another group $(2 \mathbb{Z} \imath)^{\infty}$ (all of which are defined in [5], in a less concrete fashion).

Define the following groups:

$$
\begin{aligned}
& \left((\mathbb{Z} \imath)^{\infty}=\langle\mathcal{W}\rangle,\right. \\
& \infty(\imath \mathbb{Z})=\left\langle\left\{\beta_{i} \mid i<0, i \in \mathbb{Z}\right\}\right\rangle, \\
& (\mathbb{Z} \imath)^{\infty}=\left\langle\left\{\beta_{i} \mid i \in \mathbb{N}\right\}\right\rangle .
\end{aligned}
$$

Recalling that, for instance, $W_{3} \cong\left\langle\beta_{-3}, \beta_{-2}, \beta_{-1}\right\rangle$ while $W_{2} \cong\left\langle\beta_{-2}, \beta_{-1}\right\rangle$, it is natural to think of the groups $\infty(2 \mathbb{Z})$ and $(\mathbb{Z} \imath)^{\infty}$ as limit groups built by using different families of inclusion maps $W_{i} \rightarrow W_{i+1}$.

We are now ready to state our further results.

Theorem 1.6. Suppose that $G$ is a subgroup of $\operatorname{PL}_{o}(I)$.

(1) If $G$ admits a tall tower then $G$ contains a subgroup isomorphic to $(\mathbb{Z} \imath)^{\infty}$.

(2) If $G$ admits a deep tower then $G$ contains a subgroup isomorphic to ${ }^{\infty}(2 \mathbb{Z})$.

(3) If $G$ admits a bi-infinite tower then $G$ contains a subgroup isomorphic to $(2 \mathbb{Z} 2)^{\infty}$.

The above theorem may not seem surprising, given the definitions of $\infty(\zeta \mathbb{Z})$, $(\mathbb{Z} \imath)^{\infty}$, and $(\imath \mathbb{Z} \imath)^{\infty}$ above. However, the collections of signatures from the towers in the theorem might admit other orbitals, away from the specified towers, so that the groups generated by the collections of the signatures of the towers can exhibit much more complicated behavior than can be found in $(\iota \mathbb{Z} \imath)^{\infty}$. Removing this "external complexity" is the main work of this paper. 
For any positive integer $k$, recall that we can generate $W_{k}$ using $k$ generators whose supports are aligned in a tower of $k$ single nested orbitals. If two elements of $\mathrm{PL}_{o}(I)$ have disjoint support, then recall that they must commute. In particular, the following diagram can be used to represent the group $W$. Note that it contains no infinite towers, but this realization of $W$ does admit towers of arbitrary finite height.

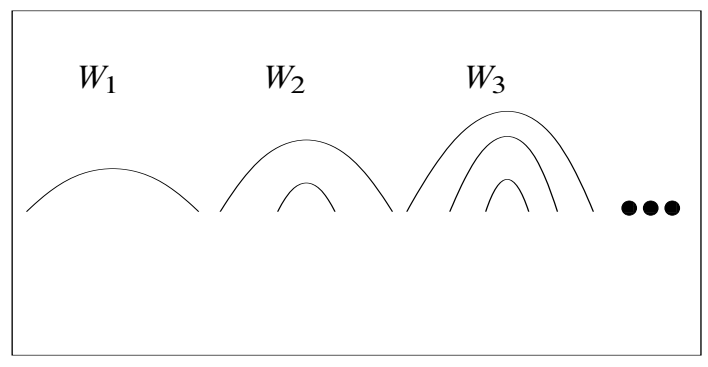

There has been some work towards further strengthening Theorem 1.1 in the case of a finitely generated non-solvable subgroup $H$ of $\operatorname{PL}_{o}(I)$. The current status (see [1]) is that one of $\infty(২ \mathbb{Z})$ or $(\mathbb{Z} \imath)^{\infty}$ must embed as a subgroup of $H$. None of that work will appear in this paper.

\section{The essential geometric theory}

In this section we will review the necessary known geometric facts about $\mathrm{PL}_{o}(I)$. We will prove only one of the results in this section, as the remainder can be found in, or are straightforward consequences of, the results in [7], [4], [3] and [2]. (While the result we do prove is new, its proof is straightforward using the ideas in [3], so we include it in this section.) For the more complex known results, we will indicate references more precisely.

2.1. The action of elements of $P_{\boldsymbol{o}}(I)$ on $I$. We begin with some often-used facts about the action of elements of $\mathrm{PL}_{o}(I)$ on the unit interval.

Remark 2.1. If $g \in \mathrm{PL}_{o}(I), A$ is an orbital of $g$ then the following holds.

(1) $g$ has finitely many orbitals.

(2) Either $x g>x$ for all $x \in A$ or $x g<x$ for all $x \in A$.

Because of the second point above, given an orbital $A$ of an element $g \in \mathrm{PL}_{o}(I)$, we will say that $g$ moves points right (left) on $A$ if $x g>x(x g<x)$ for some (and hence all) $x \in A$.

In [7] and [3] there are versions of the following lemma and its corollary, but they can also be taken as exercises for the reader. 
Lemma 2.2. Suppose that $g \in \mathrm{PL}_{o}(I)$ and $A=(a, b)$ is an orbital of $g$. Then given any $\epsilon>0$ and $x \in(a, b)$ there is an integer $n \in \mathbb{Z}$ so that the following two statements are true:

(1) $x g^{n}-a<\epsilon$, and

(2) $b-x g^{-n}<\epsilon$.

The following consequence of the above lemma can be proved by using a simple compactness argument.

Corollary 2.3. Suppose that $G \leq \mathrm{PL}_{o}(I)$ and $G$ has an orbital $A$. If $x<y$ are in $A$ then there is an element $\theta \in G$ so that $x \theta>y$.

2.2. The ubiquitous $\boldsymbol{F}$ and balanced groups. Here we mention the chief result of [4]. In essence, Brin finds that under a weak geometric condition on a subgroup $H$ of $\mathrm{PL}_{o}(I), H$ will admit an embedded copy of $F$.

If $g \in \mathrm{PL}_{o}(I)$ and $A=(a, b)$ is an open interval in $I$, then we will say that $g$ has an orbital that shares an end with $A$ if $g$ has an orbital of the form $(a, c)$ or $(c, b)$. We will also say that $g$ realizes an end of $A$ in these situations.

The main result of [4] is given below.

Theorem 2.4 (Ubiquitous $F$ ). If $H \leq \mathrm{PL}_{o}(I)$ and $H$ has an orbital $A$ so that some element $h \in H$ realizes one end of $A$, but not the other, then $H$ contains a subgroup isomorphic to Thompson's group $F$.

We will say a subgroup $G$ of $\mathrm{PL}_{o}(I)$ is balanced (following the language in [2]) if it has no subgroup $H$ which satisfies the hypotheses of Theorem 2.4. We will see some properties of balanced groups shortly.

2.3. Conjugation, orbitals, slopes, and realizations. For the statement of the next lemma to make sense, we need more definitions. If $g \in \mathrm{PL}_{o}(I)$, we will establish the notation:

$$
\mathcal{B}(g)=\left\{x \in(0,1) \mid g^{\prime}(x) \text { does not exist }\right\},
$$

that is, $\mathcal{B}(g)$ is the set of points in $(0,1)$ where the derivative of $g$ is undefined. We will call the components of $[0,1] \backslash \mathscr{B}(g)$ the affine components of $g$ (note that these are simply subsets of the domain of $g$ over which $g$ is affine). If $A$ is a connected subset of $[0,1]$ and $C$ is an affine component of $g$, then we will call $C \cap A$ an affine component of $g$ in $A$. Observe that the affine components of $g$ are finite in number and come in a natural left-to-right order which we will use naturally in our language. The following is a restatement of Remark 2.1 in [3].

Lemma 2.5. Suppose that $g, h \in \mathrm{PL}_{o}(I)$ and $A$ is an orbital of $g$. The following holds: 
(1) $g^{h}$ has orbital $A h=\{a h \mid a \in A\}$.

(2) If $g$ moves points right (resp. left) on A, then $g^{h}$ moves points right (left) on Ah.

(3) The slope of $g$ on the leftmost (resp. rightmost) affine component of $g$ in A equals the slope of $g^{h}$ on the leftmost (rightmost) affine component of $g^{h}$ in Ah.

The following diagram demonstrates this behavior; recall that "up bumps" in diagrams represent regions where the graph of a function is above the line $y=x$. In particular, the function $h$ below throws points to the right in its support.

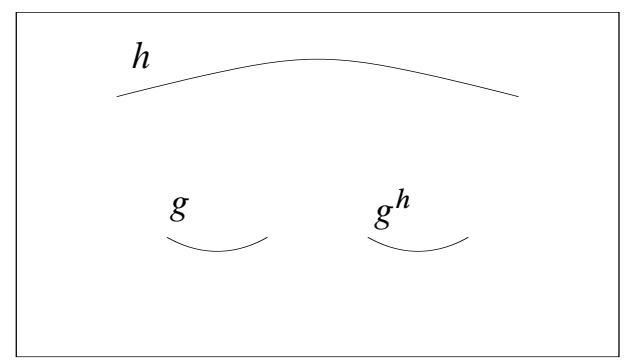

Note that it is a straightforward consequence of the previous lemma that the orbitals of $g$ and $g^{h}$ are in one-to-one ordered (left to right) correspondence. In general, we will refer to the orbital $A h$ as the orbital of $g^{h}$ induced from $g$ by the action of $h$ (or by other similar language). We will use conjugation to find new elements with better placed supports so often in the remainder that we will generally not explicitly reference Lemma 2.5 at those times, assuming instead that its content is recalled by the reader without need of further reference.

Suppose that $G$ is a subgroup of $\operatorname{PL}_{o}(I)$ and $A=(a, b)$ is an orbital of $G$, further suppose that there is an element $g \in \mathrm{PL}_{o}(I)$ that realizes both ends of $A$. Suppose that the slope of $g$ on the leftmost affine component of $g$ which non-trivially intersects $A$ is $s_{l}$, while the slope of $g$ on the rightmost affine component of $g$ which non-trivially intersects $A$ is $s_{r}$. We say that $g$ realizes $A$ inconsistently if $s_{l}$ and $s_{r}$ are either both greater than one or both less than one. Otherwise, we say that $g$ realizes $A$ consistently. We may on occasion say that " $g$ realizes the ends of $A$ consistently (or inconsistently)". If $g$ has $A$ as an orbital, then we say that $g$ realizes $A$ (note that in this case, $g$ realizes $A$ consistently).

We will now mention some promised properties of balanced subgroups of $\mathrm{PL}_{o}(I)$. The interested reader is encouraged to examine Section 3.3 of [2]. The following lemma lists two straightforward consequences of the discussion there.

Lemma 2.6. Suppose that $G$ is a balanced subgroup of $\mathrm{PL}_{o}(I)$ and $G$ has an orbital $A$ and an element $g$ which realizes both ends of $A$.

(1) $g$ realizes both ends of $A$ consistently, then $g$ realizes $A$.

(2) If $g$ realizes $A$ inconsistently, then no element of $G$ realizes A consistently. 
Suppose that $S$ is a balanced subgroup of $\mathrm{PL}_{o}(I)$ and $A$ is an orbital of $G$. By the lemma above, either every element of $G$ which realizes the ends of $A$ realizes $A$, or no element of $G$ which realizes the ends of $A$ realizes $A$. In the first case, we call $A$ a consistent orbital of $G$, in the second case we call $A$ an inconsistent orbital of $G$.

2.4. More on towers, transition chains, balanced groups, and solvability. Given a tower $T$ for a group $G \leq \mathrm{PL}_{o}(I)$, we may pass to the group $\left\langle S_{T}\right\rangle$ generated by the signatures of the tower. This group can be fairly complicated, depending in part on how the other orbitals of the signatures of $T$ align with each other. We will say a tower $T$ is an exemplary tower if whenever $(A, g),(B, h) \in T$ with $A \neq B$ then $(A, g) \leq(B, h)$ implies both

(1) the orbitals of $g$ are disjoint from the ends of the orbital $B$, and

(2) no orbital of $g$ in $B$ shares an end with $B$.

Note that this definition says something much stronger than the fact that the closure of $A$ is in $B$; it says that no orbitals of $g$ have support inside of a small neighborhood of the ends of $B$ in $B$.

The following is a conglomeration of results from [3] (Remark 2.9, Lemma 2.12, Lemma 3.2, and Remark 4.1) with a straightforward extension of Lemma 3.14.1 in [2]. We will refer to it often in the remainder.

Lemma 2.7. Suppose that $G$ is a subgroup of $\mathrm{PL}_{o}(I)$ that fails to admit transition chains of length two. We have the following consequences:

(1) $G$ is balanced.

(2) If $T$ is a tower for $G$, then $T$ is exemplary.

(3) If $f, g \in G, A$ is an orbital of $f, B$ is an orbital of $g$, and $A \cap B \neq \emptyset$, then one of the following three statements holds:

(a) $A=B$, and $A$ is an orbital of $\langle f, g\rangle$.

(b) $\bar{A} \subset B, A \cap A g=\emptyset$, and $B$ is an orbital of $f g, g f$, and of $\langle f, g\rangle$.

(c) $\bar{B} \subset A, B \cap B f=\emptyset$, and $A$ is an orbital of $f g, g f$, and of $\langle f, g\rangle$.

(4) If $G$ is a subgroup of depth $n$ for some positive integer $n$, then the derived subgroup $G^{\prime}$ has depth $n-1$.

2.5. The manipulation of supports. Given a group $G \leq \mathrm{PL}_{o}(I)$, we sometimes need to understand whether an element-orbital survives as an element-orbital in the derived subgroup $G^{\prime}$.

Lemma 2.8. Suppose that $G$ is a subgroup of $\mathrm{PL}_{o}(I)$. If $T=\left\{\left(A_{1}, g_{1}\right),\left(A_{2}, g_{2}\right)\right\}$ is an exemplary tower of height two for $G$, where $\left(A_{1}, g_{1}\right) \leq\left(A_{2}, g_{2}\right)$, then there is $M \in \mathbb{N}$ so that for all $n \in \mathbb{N}$ with $n \geq M$ we have that $A_{1}$ is an orbital of the element $\left[g_{1}, g_{2}^{n}\right]$. 
Proof. Suppose that $A_{2}=(a, b)$, and let $[x, y]$ be the smallest interval so that Supp $g_{1} \cap A_{2} \subset[x, y]$. (This interval exists by the definition of an exemplary tower.) We see immediately that $A_{1} \subset[x, y]$. Let $\epsilon=\max (x-a, b-y)$. By Lemma 2.2 there is $S \in \mathbb{Z}$ so that $x g_{2}^{S}>y$. Let $M=|S|$. Note that for any $n>M$, we have $[x, y] g_{2}^{n} \cap[x, y]=\emptyset$. The lemma follows immediately.

The above lemma hints that commutators might be useful in general for finding elements within a group which have supports with nice nesting properties. The following discussion provides our chief tool for finding such elements.

Suppose that two elements $h, k \in \mathrm{PL}_{o}(I)$ have the property that whenever $A$ is an orbital of $h$ and $B$ is an orbital of $k$ so that $A \cap B \neq \emptyset$, then either $A=B, \bar{A} \subset B$, or $\bar{B} \subset A$. We say that $h, k$ are mutually efficient, or that they satisfy the mutual efficiency condition, if both (1) given any orbital $C$ of $h$ that contains the closure of an orbital of $k$, then the support of $k$ in $C$ is contained in a single fundamental domain of $h$ in $C$, and (2) whenever $D$ is an orbital of $k$ that properly contains the closure of an orbital of $h$, then the support of $h$ in $D$ is contained in a single fundamental domain of $k$ in $D$ are satisfied.

Given two mutually efficient elements $h$ and $k$ in $\operatorname{PL}_{o}(I)$, we often will form the commutator $[[h, k], k]$ (recall that we use the definition $[a, b]=a^{-1} b^{-1} a b$ for the commutator symbol), which we will refer to as the double commutator of $h$ and $k$. The following is a restatement of Lemma 4.2 in [3].

Lemma 2.9. Let $h, k \in H$, where $H$ is a subgroup of $\mathrm{PL}_{o}(I)$ with no transition chains of length two. Suppose that further that $h$ and $k$ are mutually efficient. If $f=[[h, k], k]$, then $f$ has the following properties:

(1) Every orbital of $h$ whose closure is contained in an orbital of $k$ is an orbital of $f$.

(2) Every orbital of $f$ has closure contained in an orbital ofk that contains (perhaps not properly) an orbital of $h$.

From this point forward, all results and discussion will be new.

\section{Relationships amongst the key groups}

Let us now investigate the relationships between our key groups. In the discussions below, we will call $\beta_{-1}$ the top generator of $\infty(২ \mathbb{Z})$ and $\beta_{0}$ the bottom generator of $(\mathbb{Z} \imath)^{\infty}$.

Lemma 3.1. $W$ embeds in each group in the $\operatorname{set}\left\{\left\{^{\infty}(২ \mathbb{Z}),(\mathbb{Z} \imath)^{\infty},(২ \mathbb{Z} \imath)^{\infty}, B\right\}\right.$. 
Proof. We will show that $W$ embeds in $(\mathbb{Z} \imath)^{\infty}$ and ${ }^{\infty}(\zeta \mathbb{Z})$. This will complete the proof since ${ }^{\infty}(\zeta \mathbb{Z})$ and $(\mathbb{Z} \imath)^{\infty}$ each embed in $(\zeta \mathbb{Z} \zeta)^{\infty}$ and $B$.

We first embed $W$ in $(\mathbb{Z} \imath)^{\infty}$. For each $j \in \mathbb{N}$, define

$$
\Gamma_{j}=\left\{\gamma_{i, j} \mid \gamma_{i, j}=\beta_{i}^{\beta_{j+1}}, 1 \leq i \leq j, i \in \mathbb{N}\right\} .
$$

Note that each collection $\Gamma_{i}$ generates a group isomorphic to $W_{i}$, by the argument given in the introduction after the discussion of $W_{2}$, or also by the details of Brin in [5] (re-define $\Gamma_{0}$ to be the set with only the identity element of $\left.\mathrm{PL}_{o}(I)\right)$. Further, the supports of the generators in $\Gamma_{i}$ are all disjoint from the supports of the generators in $\Gamma_{j}$ whenever $i, j \in \mathbb{N}$ with $i \neq j$. Thus, for such $i$ and $j$, the elements of $\Gamma_{i}$ commute with the elements of $\Gamma_{j}$. Hence the set

$$
\Gamma=\bigcup_{i \in \mathbb{N}} \Gamma_{i}
$$

generates a group

$$
\langle\Gamma\rangle \cong \bigoplus_{i \in \mathbb{N}} W_{i} \cong W .
$$

(Note: We will use this realization of $W$ throughout the rest of this section when we refer to our realization of $W$ in $\mathrm{PL}_{o}(I)$. When we refer to "the first $n$ summands of $W$ " we will mean the subgroup $\left\langle\bigcup_{i=0}^{n} \Gamma_{i}\right\rangle \cong \bigoplus_{i=0}^{n} W_{i}$ of $W$. Note that we are ignoring the trivial $W_{0}$ summand in our count.)

We now embed $W$ in ${ }^{\infty}(\zeta \mathbb{Z})$ in a similar fashion, finding copies of each $W_{i}$ in $\infty(\zeta \mathbb{Z})$, all of which occur with mutually disjoint supports in $I$, the union of their generators will then generate a group isomorphic to $W$. Let $i \in \mathbb{N}$ and define

$$
\Upsilon_{i}=\left\{\theta_{i, j} \mid \theta_{i, j}=\beta_{-i+j-2}^{\beta_{-1}^{i}}, 1 \leq j \leq i, j \in \mathbb{N}\right\},
$$

so that $\Upsilon_{i}$ is the collection of the $i$-th conjugates of the $i$ generators beneath $\beta_{-1}$ of the generators of ${ }^{\infty}(\imath \mathbb{Z})$. Each collection $\Upsilon_{i}$ therefore generates a group isomorphic to $W_{i}$ (re-define $\Upsilon_{0}$ to be the set containing only the identity element of $\operatorname{PL}_{o}(I)$ ), while if $i \neq j$, any generator in $\Upsilon_{i}$ has disjoint support from the generators of $\Upsilon_{j}$, so that the union

$$
\Upsilon=\bigcup_{i \in \mathbb{N}} \Upsilon_{i}
$$

has the property that

$$
\langle\Upsilon\rangle \cong \bigoplus_{i \in \mathbb{N}} W_{i} \cong W .
$$

Since $W$ can be realized as a subgroup of $\mathrm{PL}_{o}(I)$, the following lemma demonstrates that the answer to Brin's Question 1 in [5] is "No".

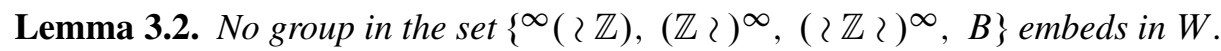


Proof. We show that neither ${ }^{\infty}(\succ \mathbb{Z})$ nor $(\mathbb{Z} \imath)^{\infty}$ embeds in $W$. This will imply that $\left(\llcorner\mathbb{Z} \imath)^{\infty}\right.$ and $B$ both fail to embed in $W$.

There is a short proof of this restricted statement using the work of Brin in [5], and our previous lemma. By our previous lemma, if $(2 \mathbb{Z})^{\infty}$ embeds in $W$, then it must embed in $(\mathbb{Z} \imath)^{\infty}$. Likewise, if $(\mathbb{Z} \imath)^{\infty}$ embeds in $W$, then it must embed in $(\iota \mathbb{Z})^{\infty}$. However, neither of these two things can occur; Theorem 2 in [5] states that the two groups $(\iota \mathbb{Z})^{\infty}$ and $(\mathbb{Z} \imath)^{\infty}$ do not embed in each other.

3.1. Transition chains and the group $B$. In this section we show that $B$ embeds in any subgroup of $\mathrm{PL}_{o}(I)$ which admits transition chains of length two.

Lemma 3.3. Suppose that $H$ is a balanced subgroup of $\mathrm{PL}_{o}(I)$ and $H=\langle\alpha, \beta\rangle$ for some two elements $\alpha, \beta \in \mathrm{PL}_{o}(I)$ and further suppose that $A$ is an inconsistent orbital of $H$ and $\alpha$ realizes both ends of $A$ while $\beta$ realizes neither. Then there is a conjugate $\gamma$ of $\beta$ in $H$ which has an orbital $B \subset A$ so that the fixed set of $\alpha$ in $A$ is contained in $B$.

Proof. Let $F_{\alpha}$ represent the fixed set of $\alpha$ in $A$, and let $x=\inf \left(F_{\alpha}\right)$ and $y=\sup \left(F_{\alpha}\right)$. Note that $x$ and $y$ are both in $A$, since $\alpha$ is non-trivial near the ends of $A$, while $F_{\alpha} \neq \emptyset$. Now by Corollary 2.3, since $A$ is an orbital of $H$, there is $\theta \in H$ so that $x \theta>y$. By the continuity of $\theta$, there is $x_{1}<x$ so that $x_{1} \theta>y$ as well. Let $z=x_{1} \theta^{-1}$ so we have

$$
z<z \theta=x_{1}<x \leq y<x_{1} \theta<x \theta .
$$

Since $A$ is an orbital of $H$, we must have that $F_{\alpha}$ is contained in the orbitals of $\beta$. In particular, we see that $\beta$ has an orbital $C=(r, s)$ so that $r<x<s$. Note further that $r \in \operatorname{Supp} \alpha \cap A$. By Lemma 2.2 there is a power $k \in \mathbb{Z}$ so that $r \alpha^{k}=q<z$. Now $\beta_{1}=\beta^{\alpha^{k}}$ has orbital $D=(q, t)$ induced from $C$ by the action of $\alpha^{k}$, and $D$ satisfies $q<z<x<t$. Set $\gamma=\beta_{1}^{\theta}$. Then $\gamma$ has orbital $B=(u, v)$ induced from $D$ by the action of $\theta$ on $\beta_{1}$, and $u=q \theta<x<y<t \theta=v$.

The following lemma is more involved and plays a key role in the proof of the lemma following immediately after. We first establish some language, parallel to language about affine components, about orbitals. Given an element $g \in \mathrm{PL}_{o}(I)$ and a set $X \subset I$, we will refer to the leftmost orbital of $g$ that is fully contained in $X$ as the leading orbital of $g$ in $X$, and the rightmost orbital of $g$ fully contained in $X$ as the trailing orbital of $g$ in $X$. We will similarly refer to the second and third such orbitals, etc., using their natural left-to-right ordering.

Lemma 3.4. Suppose that $H$ is a balanced subgroup of $\mathrm{PL}_{o}(I)$ and $H=\langle\alpha, \beta\rangle$ for some two elements $\alpha, \beta \in \mathrm{PL}_{o}(I)$. If $H$ has an inconsistent orbital $A$ and $\beta$ realizes no end of any orbital of $H$, then there are elements $\alpha_{1}$ and $\beta_{1}$ in $H$ so that if $H_{1}=\left\langle\alpha_{1}, \beta_{1}\right\rangle$ the following will be true: 
(1) $A$ is an orbital of $H_{1}$.

(2) $\beta_{1}$ realizes no end of any orbital of $H_{1}$.

(3) Every inconsistent orbital of $H_{1}$ can be written as the union of the orbitals of a transition chain of length three, whose first and last orbitals are orbitals of $\alpha_{1}$, and whose second orbital is an orbital of $\beta_{1}$.

(4) $\alpha_{1}$ moves points to the left on its leading orbital in each of the inconsistent orbitals of $H_{1}$.

Proof. We break the proof into stages, so as to make it less cumbersome.

Stage 1: Classify orbital types. Set $\alpha_{1}$ either to $\alpha$ or $\alpha^{-1}$, so that $\alpha_{1}$ moves points to the left on its leading orbital $B$ contained in $A$.

Suppose that $n \in \mathbb{N}$ and $H$ has $n$ inconsistent orbitals. Let $\mathcal{B}=\left\{B_{i} \mid 1 \leq i \leq r\right\}$ represent the collection of inconsistent orbitals of $H$ where $\alpha_{1}$ moves points to the left on its leading orbital in each of these orbitals, indexed from left to right, where $r$ is the total number of such orbitals. Let $\ell=\left\{C_{j} \mid 1 \leq j \leq s\right\}$ represent the other inconsistent orbitals of $H$, so that $n=s+r$, where these orbitals are indexed from the left to the right as before. Note that it is possible for $s=0$, although of course $r \geq 1$.

Stage 2: Building an element to span the fixed sets of $\alpha_{1}$ in the $B_{i}$. By Lemma 3.3, for each orbital $B_{i}$ in $\mathcal{B}$ there is an element $\gamma_{i}$ in $H$, which is a conjugate of $\beta$, so that the fixed set of $\alpha_{1}$ in $B_{i}$ is contained in a single orbital of $\gamma_{i}$. Likewise, for each orbital $C_{j}$ in $\ell$ there is an element $\theta_{j}$ in $H$, which is a conjugate of $\beta$, so that the fixed set of $\alpha_{1}$ in $C_{j}$ is contained in a single orbital of $\theta_{j}$.

Firstly, inductively replace each element $\gamma_{i}$, for $i>1$, by a conjugate of $\gamma_{i}$ by a high negative power of $\alpha_{1}$ so that for each $j$ with $1 \leq j<i$ the closure of the union of all of the orbitals of $\gamma_{i}$ in $B_{j}$ is actually fully contained in the single orbital of $\gamma_{j}$ that contains the fixed set of $\alpha_{1}$ in $B_{j}$. We can do this due to the specified directions in which $\alpha_{1}$ moves points on its first and last orbitals in each of the $B_{k}$.

Summing up, for each $i \in\{1,2, \ldots, r\}, \gamma_{i}$ has an orbital $D_{i}$ that contains the fixed set of $\alpha_{1}$ in $B_{i}$, as well as the closure of all of the orbitals of $\gamma_{k}$ in $B_{i}$ for all $k \in \mathbb{N}$ where the inequalities $i<k \leq r$ hold.

We will now inductively define a sequence of elements $\left(\rho_{i}\right)_{i=1}^{r}$ so that the $\rho_{i}$ will have the following properties (modulo the fact that some of the $\gamma_{i}$ below will actually be conjugates of the existing $\gamma_{i}$ by further negative powers of $\alpha_{1}$ ):

(1) $\rho_{1}=\gamma_{1}$.

(2) For all indices $i>1, \rho_{i}$ will be either a conjugate of $\rho_{i-1}$ by some power of $\gamma_{i}$, or $\rho_{i}=\rho_{i-1} \gamma_{i}$.

(3) For all indices $i, \rho_{i}$ will have an orbital $E_{i}$ in $B_{i}$ that fully contains the fixed set of $\alpha_{1}$ in $B_{i}$. 
(4) If $i<r$, the orbital $E_{i}$ of $\rho_{i}$ will contain the closures of the orbitals of $\gamma_{j}$ in $B_{i}$ for all integers $j$ with $i<j \leq r$.

(5) If $i>1$, for each integer $j$ with $1 \leq j<i, \rho_{i}$ will have $E_{j}$ as one of its orbitals.

Firstly, set $\rho_{1}=\gamma_{1}$, and $E_{1}=D_{1}$. By construction, $\rho_{1}$ satisfies the five inductive properties. If $r=1$, we are done. If not, suppose that $k$ is an integer so that $1<k \leq r$ and for all $i \in\{1,2, \ldots, k-1\}$ we have that $\rho_{i}$ is defined and satisfies the five defining properties of the induction. Our analysis now breaks into two cases.

If $\rho_{k-1}$ has an orbital $F_{k}$ containing either end of $D_{k}$, then there is some integer $j$ so that $\rho_{k}=\rho_{k-1}^{\gamma_{k}^{j}}$ will have orbital $E_{k}$ induced from $F_{k}$ by the action of $\gamma_{k}^{j}$ so that $E_{k}$ will contain the fixed set of $\alpha_{1}$ in $B_{k}$, as well as the closure of all of the orbitals of $\gamma_{j}$ in $B_{k}$ for integers $j$ where the inequalities $k<j \leq r$ hold.

If $\rho_{k-1}$ does not have an orbital $F_{k}$ containing either end of $D_{k}$, then we have to handle the case where $\rho_{k-1}$ has orbitals in $D_{k}$ that share ends with $D_{k}$ separately before continuing.

If $\rho_{k-1}$ has orbitals in $D_{k}$ that share ends with $D_{k}$, then replace $\gamma_{k}$ and all later $\gamma_{j}$ with conjugates of these elements by a high negative power of $\alpha_{1}$ so that $D_{k}$ either has an end contained in an orbital of $\rho_{k-1}$, or shares no end with an orbital of $\rho_{k-1}$, and repeat the whole inductive definition of $\rho_{k}$.

If $\rho_{k}$ is still undefined, then set $\rho_{k}=\rho_{k-1} \gamma_{k}$. Note that since $\rho_{k-1}$ has no orbitals in $D_{k}$ that share ends with $D_{k}$, the product $\rho_{k}=\rho_{k-1} \gamma_{k}$ realizes both ends of $D_{k}$ consistently, and therefore realizes $D_{k}$ consistently since $H$ is balanced. Therefore define $E_{k}=D_{k}$ and note that $\rho_{k}$ actually has $E_{k}$ as an orbital.

At this point $\rho_{k}$ and $E_{k}$ are both defined, and we can continue with our main argument. Note that $E_{k}$ contains the closure of all of the orbitals of all of the $\gamma_{i}$ for $i>k$, and that for each integer $j$ in $1 \leq j \leq k-1$, the closure of the orbitals of $\gamma_{k}$ in $B_{j}$ are fully contained in the orbital $E_{j}$, so that by Lemma $2.6, \rho_{k}$ will have $E_{j}$ as an orbital as well. Now by construction, $\rho_{k}$ satisfies the five defining properties of the induction.

We now examine the element $\rho_{r}$. Observe that $\rho_{r}$ contains an orbital $E_{k}$ in each $B_{k}$ where the fixed set of $\alpha_{1}$ in $B_{k}$ is fully contained in $E_{k}$. Now $\rho_{r}$ is constructed as a sequence of products using various $\gamma_{i}$ 's and conjugates of $\gamma_{i}$ 's, so $\rho_{r}$ realizes no end of any orbital of $H$ but is an element of $H$.

Stage 3: Building an element to span the fixed sets of $\alpha_{1}$ in the $C_{j}$. In an entirely analogous fashion, if $s>0$, then we can find one element $\psi_{s}$ in $H$ which realizes no end of any orbital of $H$ and which contains an orbital $F_{i}$ in each $C_{i} \in \mathcal{C}$ which contains the fixed set of $\alpha_{1}$ in that $C_{i}$.

Stage 4: Modifying our first element so that it creates no transition chains with $\alpha_{1}$ over the $C_{i}$. There is a positive integer $p$ so that $\rho=\rho_{r}^{\alpha_{1}^{p}}$ has the following two properties: 
(1) For each integer $i \in\{1,2, \ldots, r\}$, the closure of the orbitals of $\psi_{s}$ in $B_{i}$ is actually contained in the orbital $G_{i}$ of $\rho$ induced from $E_{i}$ by the action of $\alpha_{1}^{p}$.

(2) For each integer $i \in\{1,2, \ldots, s\}$, the closure of the orbitals of $\rho$ in $C_{i}$ is actually contained in the orbital $F_{i}$ of $\psi_{s}$.

This follows since for each orbital $B_{i}$ of $\mathcal{B}$, the lead orbital of $\alpha_{1}$ in $B_{i}$ has the property that $\alpha_{1}$ is moves points to the left there (and therefore moves points to the right on the trailing orbital of $\alpha_{1}$ in $B_{i}$ ), and for each orbital $C_{i}$ in $\ell$, the lead orbital of $\alpha_{1}$ in $C_{i}$ has the property that $\alpha_{1}$ is moving points to the right there (and therefore $\alpha_{1}$ moves points to the left on its trailing orbital in $C_{i}$ ).

We note in passing that the orbitals $G_{i}$ of $\rho$ contain the orbitals $E_{i}$ of $\rho_{r}$ and therefore the fixed set of $\alpha_{1}$ in the $B_{i}$.

Now there is a power $q$ of $\psi_{s}$ so that the element $\beta_{1}=\rho^{\psi_{s}^{q}}$ will have the following nice properties:

(1) For each integer $i \in\{1,2, \ldots, s\}$, the orbitals of $\beta_{1}$ in $C_{i}$ have trivial intersection with the fixed set of $\alpha_{1}$ in $C_{i}$.

(2) For each integer $i \in\{1,2 \ldots, r\}, \beta_{1}$ will have the orbital $G_{i}$ which contains the fixed set of $\alpha_{1}$ in $B_{i}$.

The first property follows since the orbitals of $\rho$ in the $C_{i}$ are contained in the orbitals $F_{i}$ of $\psi_{s}$, and so the conjugation of $\rho$ by a high power of $\psi_{s}$ will throw these orbitals off of the fixed set of $\alpha_{1}$ in the $C_{i}$. The second property follows since the orbitals of $\psi_{s}$ are fully contained in the orbitals $G_{i}$ of $\rho$ in the $B_{i}$, so that conjugation of $\rho$ by $\psi_{s}$ to any power will not change these orbitals.

It is now straightforward to check that the group $H_{1}=\left\langle\alpha_{1}, \beta_{1}\right\rangle$ satisfies all of the properties promised in the statement of the lemma.

We are now ready to prove our chief geometric result.

Theorem 1.4. If $G$ admits a transition chain of length two, then $B$ embeds in $G$.

Proof. We can assume that $G$ is balanced; otherwise by Theorem 2.4 the group $G$ will contain a copy of Thompson's group $F$, which contains copies of Brin's group $B$.

Let $\mathcal{T}=\left\{\left(O_{1}, \alpha\right),\left(O_{2}, \beta\right)\right\}$ be a transition chain of length two for $G$ and let $K=\langle\alpha, \beta\rangle$.

The orbitals of $K$ are the components of the union of the orbitals of $\alpha$ and $\beta$. Note that the consistent orbitals of $K$ (if they exist) will be realized by at least one of $\alpha$ and $\beta$. The inconsistent orbitals of $K$ are formed by the union of a sub-collection of orbitals of $\alpha$ and orbitals of $\beta$. A chief feature of the inconsistent orbitals of $K$ is that while neither $\alpha$ nor $\beta$ realize any of them, at least one of $\alpha$ or $\beta$ must realize both ends of any particular such orbital, since $K$ is balanced. Note in passing that the orbital of $K$ which contains $O_{1}$ is inconsistent, as neither $\alpha$ nor $\beta$ realize that whole orbital, although one of $\alpha$ or $\beta$ realizes both ends of it. 
We will proceed through the remainder of the proof in stages.

Stage 1: Classifying the orbitals of $K$. We are going to analyze the orbitals of $K$ still further. Any particular orbital of $K$ has one of six types, the first three are consistent, and the last three are inconsistent:

(1) (Type $\mathrm{AB}$ ) Both $\alpha$ and $\beta$ consistently realize this orbital.

(2) (Type $\mathrm{Ab}$ ) $\alpha$ consistently realizes this orbital, but not $\beta$.

(3) (Type aB) $\beta$ consistently realizes this orbital, but not $\alpha$.

(4) (Type $\underline{a} \underline{b}$ ) Both $\alpha$ and $\beta$ inconsistently realize both ends of this orbital.

(5) (Type $a b$ ) $\alpha$ inconsistently realizes both ends of this orbital, but $\beta$ realizes neither end of this orbital.

(6) (Type ab) $\beta$ inconsistently realizes both ends of this orbital, but $\alpha$ realizes neither end of this orbital.

The diagram below demonstrates a representative $\alpha$ and $\beta$ pair, and orbitals of the group $\langle\alpha, \beta\rangle$ of all six types. The group orbitals are given as heavy horizontal line segments, and they are labeled with their type. The orbitals of different types themselves come in different flavors, as will become apparent below.

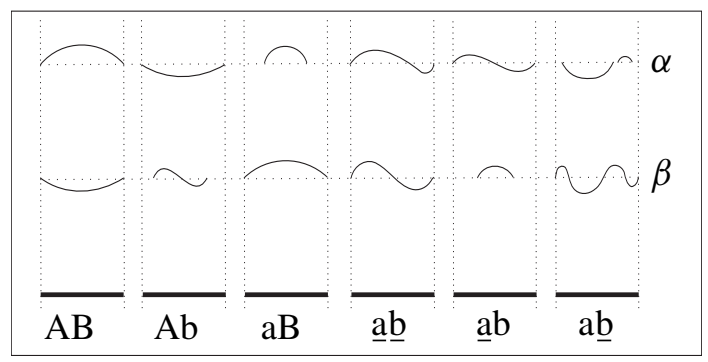

We know that $K$ has at least one orbital, let us call it $A$, of type $\underline{a} \underline{b}$, ab, or a므, and we will assume without meaningful loss of generality that $A$ has one of the first two types.

We will carry out operations on our generators which will change the orbitals and their types below. The orbitals of type a $\underline{b}$ will need particular attention; they evolve in myriad ways, depending on their individual flavors.

Let $F_{a}$ represent the union of the fixed sets of $\alpha$ that are contained in the orbitals of $K$ of type a $\underline{a}$, type $\underline{a} b$, and $a \underline{b}$. Then $F_{a}$ is non-empty and is entirely contained in the orbitals of $\beta$.

Stage 2: A mechanism for modifying elements and orbitals. By Remark 2.2 there is an $N_{1} \in \mathbb{N}$ so that for all $k \in \mathbb{N}$ with $k \geq N_{1}$ we have $F_{a} \beta^{k} \cap F_{a}=\emptyset$ in orbitals of type a $\underline{a}$ and $\underline{a} b$ (also assume that $N_{1}$ is large enough so that if $C$ is an orbital of type ab, the union of the components of $F_{a} \cap C$ which do not share an end with $C$ is moved off of itself under the action of $\beta^{k}$ ). Similarly, let $S$ represent the support 
of $\alpha$ in the orbitals of $K$ of type aB, then there is $N_{2} \in \mathbb{N}$ so that for all $k \geq N_{2}$, we have $S \beta^{k} \cap S=\emptyset$. Let $N=\max \left(N_{1}, N_{2}\right)$.

Considering the other direction, let $F_{b}$ represent the fixed set of $\beta$ in the orbitals of $K$ of type ab. Since $F_{b}$ is contained in the support of $\alpha$ by definition, there is $M \in \mathbb{N}$ so that for all $j \geq M$ we have that $F_{b} \alpha^{j} \cap F_{b}=\emptyset$.

Now let $j \geq M$, let $k \geq N$ and define $\beta_{1}=\left[\alpha^{j}, \beta^{k}\right]$.

Stage 3: Analyzing how $\beta_{1}$ differs from $\beta$. Observe that the fixed set of $\alpha$ in the orbitals of $K$ of type $\underline{a} \underline{b}$ and $\underline{a} b$ is contained in the orbitals of $\beta_{1}$. Also if $C$ is an orbital of $K$ of type ab, then the components of $F_{a} \cap C$ which are in the orbitals of $\beta$ that do not share an end with $C$ are all contained in the support of $\beta_{1}$. The fixed set of $\beta$ contained in the orbitals of $K$ of type $a \underline{b}$ is also contained in the support of $\beta_{1}$, since any such point is moved off of $F_{b}$ by $\alpha^{-j}$, then moved by $\beta^{-k}$, then moved to someplace different (from its start) by $\alpha^{j}$, and finally, $\beta^{k}$ cannot move the resultant point to its original location in the fixed set of $\beta$. Now observe that the orbitals of $\beta_{1}$ are either disjoint from $S$, or else are components of $S$ where $\alpha^{j}$ behaves as the inverse of $\beta_{1}$.

The diagram below gives a rough model of four orbitals of type ab, which type will feature heavily in the following discussion. It gives a rough estimate of the elements $\alpha^{-j}, \alpha^{\beta^{k}}$, and $\beta_{1}=\left[\alpha^{j}, \beta^{k}\right]=\alpha^{-j} \cdot \alpha^{j \beta^{k}}$ for these elements.

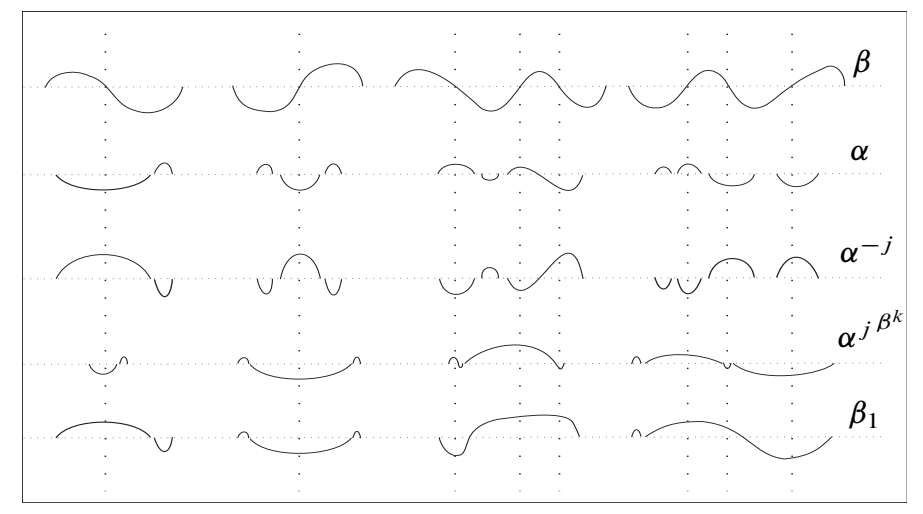

Stage 4: Analyzing the orbitals of $K_{1}$. We now consider the group $K_{1}=\left\langle\alpha, \beta_{1}\right\rangle$, and we consider the orbitals of $K_{1}$ under the same classification as the orbitals of $K$, where we replace $\beta$ by $\beta_{1}$ in that classification.

It is immediate to see that $K_{1}$ still has all the orbitals of $K$ of type $\underline{a} b$, and that the type of these orbitals is unchanged. It is also immediate by construction that the orbitals of $K$ of type $\underline{a} \underline{b}$ are also orbitals of $K_{1}$, although they are now of type $\underline{a} b$. The orbitals of $K$ of type $\mathrm{Ab}$ are also orbitals of $K_{1}$ of type Ab, but the orbitals of $K$ of type $\mathrm{aB}$ are now replaced by a collection of interior orbitals (all lying properly in the union of the orbitals of $K$ of type $\mathrm{aB}$ ), each of which is an orbital of type aB that is 
actually disjoint from the support of $\alpha$, or else of type $\mathrm{AB}$, where $\alpha^{j}$ and $\beta_{1}$ behave as inverses on these orbitals. The orbitals of $K$ of type AB are now of type Ab, and may have trivial intersection with the support of $\beta_{1}$ (if, in fact, $\alpha$ and $\beta$ commuted on these orbitals).

If $B_{1}$ is an orbital of $K$ of type ab, then $B_{1}$ is not an orbital of $K_{1}$. In this case $K_{1}$ admits a new collection of orbitals properly contained in $B_{1}$.

We first consider the case where $\beta$ is moving points to the right on its leading orbital in $B_{1}$ (and therefore is moving points to the left on its trailing such orbital). We will suppose that $k$ was chosen large enough so that the closure of the union of the orbitals of $\beta^{-k} \alpha^{j} \beta^{k}$ that are contained in orbitals of $\beta$ in $B_{1}$ is actually contained in the orbitals of $\alpha$ (and therefore of $\alpha^{j}$ ) which contain components of the fixed set of $\beta$. Note that any orbital of $\beta$ whose closure is contained in $B_{1}$ is contained in the union of the orbitals of $\alpha^{j}$ and $\beta^{-k} \alpha^{j} \beta^{k}$. Therefore, there are three possible varieties of resulting orbitals of $K_{1}$ in $B_{1}$ : firstly, of type $\mathrm{AB}$, where $\beta_{1}$ actually behaves as $\alpha^{-j}$ on these orbitals (there may be several of these), secondly, of type AB, where there is only one such orbital and it contains the fixed set of $\beta$, or thirdly, of type $\underline{a} \underline{b}$, where there is one of these if the previous variety did not occur, and it contains the fixed set of $\beta$ in this case. We will assume $k$ was chosen large enough so that these properties of transformation are preserved over all orbitals of $K$ of type ab where $\beta$ is moving points to the right on its leading relevant orbitals.

Detour: An illustrative detour. Suppose for a moment that $B_{1}$ is the third orbital of the group $\langle\alpha, \beta\rangle$, where our generators are pictured in the previous diagram. If we invert the trailing (rightmost) orbital of $\alpha$ in $B_{1}$, so that $\alpha$ throws points right in that trailing orbital, then some calculations should convince the reader that the resulting $\beta_{1}$ will have only one orbital in $B_{1}$ (over which $\beta_{1}$ would throw points to the left). Let us call this resulting orbital $C$. In this case, $C$ would be of type aB. This contradicts our description in the previous paragraph. What has happened? Well, in this case $\alpha$ realizes both ends of $C$ consistently, but has interior fixed set, thus showing that the initial group was not balanced (by Lemma 2.6).

Let us return to our main argument.

In the case of the orbitals of $K$ of type $a \underline{b}$ where $\beta$ moves points to the left on its leading relevant orbitals. The results depend heavily on the nature of $\alpha$ in these individual orbitals. To clarify the discussion, let us suppose that $B$ is such an orbital, and discuss the possibilities that arise from the behavior of $\alpha$ and $\beta$ on $B$.

Firstly, let us suppose that $\alpha$ has an orbital that contains the fixed set of $\beta$ in $B$. In this case, let us suppose that $k$ and $j$ were chosen large enough so that the entire support of $\alpha$ is contained inside a single fundamental domain of the single orbital of $\beta^{-k} \alpha^{j} \beta^{k}$ that contains the fixed set of $\beta$ in $B$. In this case, the group $K_{1}$ possibly has several orbitals in $B$, all of type aB. One of these orbitals contains all of the support of $\alpha$ in $B$, and all of the rest are orbitals of $\beta_{1}$ which contain no orbitals of $\alpha$ and are therefore of type $\mathrm{aB}$ with trivial intersection with orbitals of $\alpha$. 
Now let us suppose that $\alpha$ has more than one orbital in $B$ that contains a component of the fixed set of $\beta$. The first and last such orbitals of $\alpha$ in $B$ must have that $\alpha$ behaves inconsistently on these orbitals, otherwise it is easy to create an imbalanced subgroup of $K_{1}$. So now there are two further cases.

Let us suppose that $\alpha$ moves points to the right on its first orbital in $B$ which contains a component of the fixed set of $\beta$, and therefore moves points to the left on the last such. In this case $K_{1}$ has only one orbital in the domain $B$, call it $C$, which is again of type ab. The closure of $C$ is contained in $B$, and $\beta_{1}$ moves points to the right on its leading orbital in $C$ and moves points to the left on its trailing orbital there.

Now let us suppose that $\alpha$ moves points to the left on its leading orbital in $B$ that contains a component of the fixed set of $\beta$, and therefore moves points to the right on its trailing orbital in $B$ which contains a component of the fixed set of $\beta$, the group $K_{1}$ again has some pure orbitals (type aB) plus precisely one orbital $C$ in $B$, which is again of type $\mathrm{a} \underline{\mathrm{b}}$, and this time, $\beta_{1}$ will move points to the left on its leading orbital in $C$ and will move points to the right on its trailing orbital in $C$.

The result of all of this analysis is the following, we can choose $j$ and $k$ so that the group $K_{1}$ has orbitals of the following types:

(1) $\mathrm{AB}$

Note that in this case $\alpha$ and $\beta_{1}$ commute on this orbital, except in the case possibly generated from orbitals of type $a \underline{\mathrm{b}}$ where $b$ moves points right on its leading orbital.

(2) $\mathrm{Ab}$

(3) $\mathrm{aB}$

Note here that the behavior of $\alpha$ on this orbital is as the identity, unless this orbital is contained in an orbital of $K$ of type $a \underline{b}$, in which case $\alpha$ may have non-trivial support in this orbital.

(4) $\underline{a} \underline{b}$

Note that orbitals of this type are always contained in orbitals of $K$ of type $a \underline{b}$ where $\beta$ moves points to the right on its first relevant orbital.

(5) $\underline{\mathrm{a}} \mathrm{b}$

Since these are the certain result of an orbital of type a $\underline{a}$ or of an orbital of type ab of $K$, we see that $K_{1}$ will have at least one of these.

(6) $\mathrm{a} \underline{\mathrm{b}}$

These orbitals all have the property that whenever $\beta_{1}$ moves points to the left on its leading orbital in these orbitals, then $\alpha$ moves points to the left on its leading orbital of the orbitals that contain a component of the fixed set of $\beta_{1}$. 
Stage 5: Evolving the group $K$ and its orbitals by repeatedly applying the mechanism.

We can repeat the process above to create a new element $\beta_{2}$ using $\alpha$ and $\beta_{1}$, and therefore a new group $K_{2}=\left\langle\alpha, \beta_{2}\right\rangle . K_{2}$ improves on $K_{1}$ since all of its orbitals of type $\mathrm{a} \underline{\mathrm{b}}$ have both $\beta_{2}$ and $\alpha$ moving points to the left on their important leading orbitals. In particular, $K_{2}$ may still have orbitals of type a $\underline{a}$, and of type AB (although here $\alpha$ and $\beta_{2}$ will commute on these orbitals). $K_{2}$ may have orbitals of type $\mathrm{Ab}$, but its orbitals of type aB will all have the property that $\alpha$ is the identity over these orbitals, while $K_{2}$ will certainly have orbitals of type ab. Repeating the process one more time to create an element $\beta_{3}$ and a subgroup $K_{3}=\left\langle\alpha, \beta_{3}\right\rangle$ produces a group whose orbitals are much easier to describe. $K_{3}$ will have no orbitals of type AB since $K_{2}$ had no orbitals of type $\mathrm{aB}$ or $\mathrm{ab}$ that could produce these orbitals (the types exist, but not with the right sub-flavors of $\alpha$ and $\beta_{2}$ to generate these offspring). $K_{3}$ may have orbitals of type $\mathrm{Ab}$, but it will have no orbitals of type $\mathrm{aB}$, since the orbitals of type aB in $K_{2}$ had $\alpha$ behaving as the identity there, and $K_{2}$ had no orbitals of type ab with $\beta_{2}$ moving points to the left on its first sub-orbital $D$ while $\alpha$ was moving points to the right on its orbital containing the right end of $D . K_{3}$ will have no orbitals of type $\underline{a} \underline{b}$, since $K_{2}$ had no orbitals of type ab with $\beta_{2}$ moving points to the right on its first orbital in the orbitals of $K_{2}$ of this type. $K_{3}$ will have at least one orbital of type $\underline{a b}$, and may have several orbitals of the type ab, but all of these last will have $\beta_{3}$ moving points to the left on its leftmost orbitals in these orbitals, and $\alpha$ will also move points to the left on its first orbitals containing the right ends of $\beta_{3}$ 's leftmost orbitals in these orbitals of type ab of $K_{3}$.

Now the orbitals of $K_{3}$ are well understood, and the behaviors of $\beta_{3}$ and $\alpha$ on these orbitals are also well understood. We now consider the subgroup $K_{4}$ generated by $\alpha$ and $\beta_{4}=\left[\alpha^{-j}, \beta_{3}^{k}\right]$, where $j$ and $k$ are chosen as in the previous process (note the negative index on $\alpha$ ). The point of this is that now the orbitals of $K_{4}$ will admit no orbital of type $\mathrm{a} \underline{\mathrm{b}}$ with $\beta_{4}$ moving points to the left on its first orbital. Now replacing $K_{4}$ with $K_{5}=\left\langle\alpha, \beta_{5}\right\rangle$ where $\beta_{5}=\left[\alpha^{j}, \beta^{k}\right]$ where $j$ and $k$ are chosen as before produces a group with no orbitals of type $\mathrm{ab}$, repeating one more time to generate $\beta_{6}$ and $K_{6}$ in the same fashion that we generated $K_{1}$ from $K$ produces a group whose orbitals are only of types $\mathrm{Ab}$ and $\underline{\mathrm{ab}}$.

Let us consider the orbital $A$ of $K$. $A$ is also an orbital of $K_{6}$, and it is of type ab. We will now replace $K$ by $K_{6}$ and $\beta$ by $\beta_{6}$ so that $K$ has an orbital of type ab and all of its orbitals are of type $\underline{a} b$ and $A b$.

Stage 6: Improving the inconsistent orbitals of $K$. Suppose that $K$ has $n$ orbitals of type $\underline{\mathrm{ab}}$, and let $\mathcal{O}=\left\{A_{i} \mid 1 \leq i \leq n, i \in \mathbb{N}\right\}$ represent this collection, where the indices respect the left-to-right order of the orbitals. By construction we know that $n \geq 1$. Apply Lemma 3.4 (above) to replace $\alpha$ and $\beta$ by new elements, and replace $K$ by the new group generated by the new $\alpha$ and $\beta$ so that $\beta$ still realizes no end of any orbital of $K$, and $A_{1}$ is still an orbital of type $\underline{\mathrm{ab}}$, but where every maximal transition 
chain (of length greater than one) which can be formed by using $\alpha$ and $\beta$ has length three (naturally $\alpha$ provides the leading and trailing orbitals for any such chain), and where $\alpha$ moves points to the left on all of its leading orbitals in orbitals of type ab for $K$ (and therefore moving points to the right on its trailing such intervals).

Stage 7: Improving the consistent orbitals of $K$. We now improve $\beta$ so that it will not admit support in the orbitals of $K$ of type Ab.

Choose two integers $m$ and $n$ intelligently. Choose $n$ large enough so that the entire support of $\beta$ in the inconsistent orbitals of $K$ is contained in the set of orbitals of $\beta^{\alpha^{n}}$ which contain the fixed sets of $\alpha$ in these inconsistent orbitals, and so that in the consistent orbitals of $K$, the conjugate $\beta^{\alpha^{n}}$ has support disjoint from the support of $\beta$, being entirely to the left (or entirely to the right) of the support of $\beta$ in each of these individual orbitals. Choose $m$ large enough so that the support of $\beta$ in the orbitals of $\beta^{\alpha^{n}}$ (in the inconsistent orbitals of $K$ ) is moved entirely to the right of itself (or entirely to the left of itself, depending on the orbital of $\beta^{\alpha^{n}}$ involved) by the action of $\left(\beta^{m}\right)^{\alpha^{n}}$.

Replace $\beta$ by the commutator $\left[\beta^{m},\left(\beta^{m}\right)^{\alpha^{n}}\right]$. The new beta still has a single orbital spanning the fixed set of $\alpha$ in each of the inconsistent orbitals of the original $K$. Also, the new $K$ generated by $\alpha$ and the new $\beta$ has the same orbitals as the previous $K$, except now the support of $\beta$ is contained in the inconsistent orbitals of $K$. In particular, the consistent orbitals of $K$ now only support the action of powers of $\alpha$.

Stage 8: Finding $B$ in $K$. Define $\tilde{\gamma}_{0}=\beta$. There is a natural number $k$ so that $\tilde{\gamma}_{1}=\gamma_{0}^{\alpha^{k}}$ has the property that if $Z$ is any particular orbital of $K$ of type ab. Then the closure of the support of $\gamma_{0}$ in $Z$ is fully contained in the orbital of $\tilde{\gamma}_{1}$ that contain the fixed set of $\alpha$ in $Z$. Note that this $k$ exists, since $\alpha$ moves points to the left on all of its leading orbitals in the orbitals of $K$ of type ab. Replace $\alpha$ by $\alpha^{k}$.

There is another natural number $j$ so that the closure of the support of $\tilde{\gamma}_{0}$ in any particular orbital $Y$ of $\tilde{\gamma}_{1}$ is fully contained in a single fundamental domain of $\tilde{\gamma}_{1}^{j}$ in $Y$. In particular, if we replace $\gamma_{i}$ by $\left(\gamma_{0}^{j}\right)^{\alpha^{i}}$ for every $i \in \mathbb{Z}$, then the group generated by the new set of $\gamma_{i}$ will be isomorphic with $(2 \mathbb{Z} \imath)^{\infty}$ since the support of each such $\gamma_{i}$ in any orbital of type $\mathrm{ab}$ of $K$ is wholly contained inside a single fundamental domain of an orbital of $\gamma_{i+1}$. Since the orbitals of $\alpha_{i}$ which create the consistent orbitals of $K$ do not effect the isomorphism type of the group $\left\langle\alpha, \gamma_{0}\right\rangle$ (being disjoint from the support of $\left.\gamma_{0}\right)$, we see that $\left\langle\alpha, \gamma_{0}\right\rangle \cong B$.

3.2. Finding infinite wreath products in groups with infinite towers. Suppose that $D=\left\{\left(A_{k}, h_{k}\right) \mid k \in \mathbb{N}\right\}$ is an exemplary tower whose indexing respects the order of the elements. Suppose further that $D$ has the property that whenever $B$ is an orbital of $h_{k}$ for some signature $h_{k}$ of $D$, then $B$ is contained in an orbital $C$ of $h_{k+1}$.

We are going to find a sub-tower of $D$ that satisfies a convenient further property.

Suppose that $k \in \mathbb{N}$ and $B_{k}$ is an orbital of $h_{k}$, but possibly $B_{k} \neq A_{k}$. By the definition of $D$, each signature $h_{j}$ of $D$ has an orbital $B_{j}$ that contains $B_{k}$ for $j>k$. 
The orbitals $B_{j}$ are nested as the index $j$ increases, but possibly not properly. If there is an $N \in \mathbb{N}$ so that for all $n \geq N \geq k$, we have $B_{n}=B_{n+1}$, then we will call $B_{k} a$ terminal orbital of $h_{k}$ with respect to $D$, and $\left(B_{k}, h_{k}\right)$ a terminal signed orbital with respect to $D$, and we will say that $B_{k}$ is stable after $N$ with respect to $D$. We will also call any orbital of a signature of the tower $D$, where the orbital is not a terminal orbital for that signature with respect to $D$, a non-terminal orbital (note that in this language the tower and signature will then be understood by context). In similar fashion, we will allow ourselves to drop portions of the phrase "of $h_{i}$ with respect to the tower $D$ " in any reference to terminal orbitals, if the tower or signature is easily understood from the context.

Observe that non-terminal signed orbitals make good candidates for being bases of new exemplary towers.

We will rely heavily on the following result in our proof of Lemma 3.6. Given an infinite exemplary tower satisfying the orbital-containment property in the first paragraph of this section, the lemma below allows us to pass to an infinite sub-tower where all non-terminal signed orbitals form bases of new infinite exemplary towers (using the same signatures (after a certain index) as in the initial tower).

Whenever we apply the results of the upcoming lemma to improve a tower, we will state that we are employing a growing tower operation.

Lemma 3.5 (Growing tower). Suppose that $D=\left\{\left(A_{k}, h_{k}\right) \mid k \in \mathbb{N}\right\}$ is an exemplary tower so that whenever $B$ is an orbital of $h_{k}$ for some index $k$, then $B$ is contained in an orbital C of $h_{k+1}$. Then we can pass to an infinite sub-tower $E=\left\{\left(B_{k}, g_{k}\right) \mid k \in \mathbb{N}\right\}$ of $D$ so that if $J$ is an orbital of any signature $g_{k}$ of $E$, where $J$ is a non-terminal orbital with respect to $D$, there is an orbital $K$ of $g_{k+1}$ which properly contains $J$.

Proof. In the discussion below, we will pass repeatedly to infinite sub-towers of $D$, at each stage referring to the new tower as $D$, and re-indexing so that the new tower will still be described as $D=\left\{\left(A_{k}, h_{k}\right) \mid k \in \mathbb{N}\right\}$.

Our argument will require a double induction. We will induct across a set of pairs $(i, k)$ of indices. The index $k$ will be called the vertical index and will represent the $k$-th "level" of the tower $D$ (i.e., with signature $h_{k}$ ), and the index $i$ will be called the horizontal index and will progress from left to right across the $n_{k}$ non-terminal orbitals of $h_{k}$ with respect to $D$.

For each vertical index $k$, starting at $k=0$, we progress across the relevant horizontal index, improving the tower $D$ for each $i$ with $1 \leq i \leq n_{k}$. Our new tower $D$ will be similar to the old $D$, except that we will have removed many of the old "levels" above the index $k$ (while still leaving and infinite tower). After re-indexing, the values of the $n_{j}$ 's may have changed for $j>k$.

Having improved $D$ at the vertical stage $k$, we will progress to the $k+1$ stage, and repeat the process there. We now define the process at index $k=0$. 
Let $\mathcal{P}=\left\{C_{i, 0} \mid 1 \leq i \leq n_{0}, i \in \mathbb{N}\right\}$ represent the $n_{0}$ orbitals of $h_{0}$ that are non-terminal with respect to $D$, in left-to-right order. First, we will improve $D$ by passing to an infinite sub-tower $n_{0}$ times.

For $C_{1,0}$, consider the sequence $\left(\left(E_{1,0, j}, h_{j}\right)\right)_{j=0}^{\infty}$ of signed orbitals where for each index $j>0$ we have that $E_{1,0, j-1} \subset E_{1,0, j}$, and where $E_{1,0,0}=C_{1,0}$. Define a function $\sigma_{1,0}: \mathbb{N} \rightarrow \mathbb{N}$ which takes value 1 at $j>0$ if $E_{1,0, j}=E_{1,0, j-1}$, and 0 otherwise. Note that $\sigma_{1,0}$ takes on the value 0 infinitely often, since $C_{1,0}$ is not a terminal orbital, and also note that $\sigma_{1,0}(0)=0$. Pass to the sub-tower of $D$ which consists precisely of the signed orbitals that had an index $j$ where $\sigma_{1,0}(j)=0$. This new version of $D$ has the following properties:

(1) The new signature $h_{0}$ is the same signature as $h_{0}$ in the previous $D$,

(2) $D$ is infinite, and

(3) for every index $k>0$, the orbital of $h_{k}$ containing $C_{1,0}$ properly contains the orbital of $h_{k-1}$ containing $C_{1,0}$.

Now repeat the process above for each horizontal index $i$ from 2 to $n_{0}$, where at each $i$, the sequence $\left(\left(E_{i, 0, j}, h_{j}\right)\right)_{j=0}^{\infty}$ of signed orbitals is built using the conditions that $E_{i, 0,1}=C_{i, 0}$ and $E_{i, 0, j} \subset E_{i, 0, j+1}$ for each index $j$. This will result in a new tower $D$ which satisfies the following properties:

(1) The new signature $h_{0}$ is the same signature as $h_{0}$ in the initial $D$,

(2) the new $D$ is still infinite,

(3) for every vertical index $k>0$ and for each $i$ with $1 \leq i \leq n_{1}$, the orbital of $h_{k}$ containing $C_{i, 0}$ properly contains the orbital of $h_{k-1}$ containing $C_{i, 0}$.

In particular, each of the signed orbitals $\left(C_{i, 0}, h_{0}\right)$ could be used as the lowest level of an exemplary tower using the signatures $h_{k}$.

We will now induct up the vertical indices $k$, applying the same process at each new value of $k$ as we did when $k$ was zero.

At the $k$-th stage of the vertical induction, the signature $h_{k}$ is preserved in the new tower, and only higher "levels" are deleted. In particular, at each index $k$, the new tower $D$ is stable below index $k+1$ for the remainder of the process. Thus, this process results in a new infinite tower $D$, and that tower has the properties claimed in the lemma statement (at any vertical "level" $k$, all non-terminal orbitals of $h_{k}$ with respect to $D$ have the property that they are properly contained in non-terminal orbitals of $h_{k+1}$ ).

The diagram below demonstrates the process of deleting different levels of a tower satisfying the orbital-containment hypothesis of this section. The dashed horizontal lines indicate a deletion and are labeled with the index pair $(k, i)$ of the double induction in the proof above that resulted in the deletion of that level. 


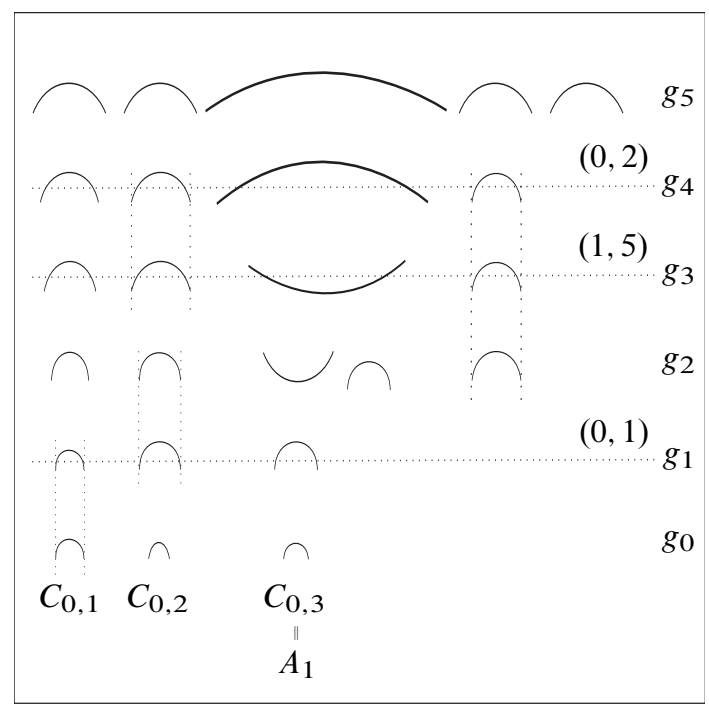

The following lemmas are simply restatements (with proofs) of the different aspects of Theorem 1.6.

Lemma 3.6. If $G$ is a subgroup of $\mathrm{PL}_{o}(I)$ and $G$ admits a tall tower, then $G$ has a subgroup of the form $(\mathbb{Z} 2)^{\infty}$.

Proof. We again proceed in stages.

Stage 1: Making observations which enable a simplified treatment of orbitals. We will assume that $G$ admits no transition chains of length two, as otherwise Theorem 1.4 will imply that $G$ contains an embedded copy of $B$ which contains isomorphic copies of $(Z \text { 乙 })^{\infty}$.

Stage 2: Choosing an initial tower and noting its supporting orbital. Let $E_{0}=$ $\left\{\left(A_{i}, g_{i}\right) \mid i \in \mathbb{N}\right\}$ be a tall tower for $G$, where the indexing respects the order on the signed orbitals of $E_{0}$. By Lemma 2.7, since $G$ contains no transition chains of length two, $E_{0}$ is exemplary.

Let $A=\bigcup_{i \in \mathbb{N}} A_{i}=(a, b)$. We observe that if $B$ is an orbital of $g_{i}$ for some $i$, then $B$ is disjoint from $\{a, b\}$. In particular, $A$ is an orbital of $\left\langle S_{E_{0}}\right\rangle$.

Stage 3: Improving our tower inside of the supporting orbital $A$. Now given $\epsilon>0$ so that $\epsilon<\frac{b-a}{2}$, we see that there is a least $N_{\epsilon} \in \mathbb{N}$ so that for all $n \in \mathbb{N}$ with $n \geq N_{\epsilon}$, we have that $(a+\epsilon, b-\epsilon) \subset A_{n}$, as the ends of the $A_{i}$ must limit to the ends of $A$. Let us construct an order-preserving function, $\phi: N \rightarrow N$, so that given any $n \in \mathbb{N}$, all of the orbitals of $g_{n}$ in $A$ are actually contained in $A_{\phi(n)}$. Since $E_{0}$ is exemplary, if $\phi(n) \neq n$ then no orbital of $g_{n}$ in $A$ actually shares an end with $A_{\phi(n)}$.

For any $k \in \mathbb{N}$, let $\phi^{k}$ represent the product (via composition) of the function $\phi$ with itself $k$ times in the monoid of order-preserving functions from $\mathbb{N}$ to $\mathbb{N}$ (use 
$\phi^{0}=\mathrm{id}$, the function which moves nothing). Now define an order-preserving function $\theta: \mathbb{N} \rightarrow \mathbb{N}$, given by the rule $n \mapsto \phi^{n}(0)$ for each $n \in \mathbb{N}$. Create $E_{1}$, the exemplary tower formed by the collection $\left\{\left(A_{\theta(i)}, g_{\theta(i)}\right) \mid i \in \mathbb{N}\right\}$. To simplify the discussion below, re-index and relabel the signatures of the tower $E_{1}$ in the natural, orderpreserving fashion, so that we will again denote these signed orbitals as $\left(A_{i}, g_{i}\right)$. We thus write $E_{1}=\left\{\left(A_{i}, g_{i}\right) \mid i \in \mathbb{N}\right\}$.

$E_{1}$ now has the property that if $i, k \in \mathbb{N}$ with $i<k$ then all the orbitals of $g_{i}$ in $A$ are actually in $A_{k}$, away from the ends of $A_{k}$. For each $n \in \mathbb{N}$, with $n>0$, let $m_{n}$ be an integer large enough so that the collection of orbitals of $g_{n-1}$ inside of $A_{n}$ (which is all the orbitals of $g_{n-1}$ in $A$ ) is actually fully contained in a single fundamental domain of $g_{n}^{m_{n}}$ in $A_{n}$. Define $m_{0}=1$. Improve $E_{1}$ to create a new tower $E_{2}$ by replacing each signature $g_{n}$ with $g_{n}^{m_{n}}$ (and re-index and relabel its signed orbitals as above, so that we will again write $\left.E_{2}=\left\{\left(A_{i}, g_{i}\right) \mid i \in \mathbb{N}\right\}\right)$. Now by construction, given any index $k$, all the orbitals of $g_{k}$ which are contained inside of $A$ are actually contained inside a single fundamental domain of $g_{k+1}$ in $A_{k+1}$.

Stage 4: Improving our tower outside of the orbital A. We cannot immediately pass to a growing sub-tower; some work needs to be done to $E_{2}$ in order for it to satisfy the hypotheses of Lemma 3.5.

Define $h_{0}=g_{0}$. Now for each $n \in \mathbb{N}$ with $n>0$, inductively define $h_{n}$ via the following process.

Let $\left\{B_{n, 1}, B_{n, 2}, \ldots, B_{n, j}\right\}$ represent the orbitals of $g_{n}$ that are also orbitals of $h_{n-1}$. Let $s_{g, n, k, l}$ and $s_{g, n, k, r}$ represent the leftmost and rightmost slopes of $g_{n}$ on the orbital $B_{n, k}$, while $s_{h, n, k, l}$ and $s_{h, n, k, r}$ represent the leftmost and rightmost slopes of $h_{n-1}$ on $B_{n, k}$, for each index $k$. It is immediate that there is a least positive integer $r_{n}$ so that for each index $k$ we have that $s_{h, n, k, l} \cdot s_{g, n, k, l}^{r_{n}} \neq 1$ and $s_{h, n, k, r} \cdot s_{g, n, k, r}^{r_{n}} \neq 1$.

Define $h_{n}^{\prime}=h_{n-1} g_{n}^{r_{n}}$. By the discussion in the previous paragraph, we see that $h_{n}^{\prime}$ is not the identity in $B_{n, k}$ near the ends of $B_{n, k}$ for each index $k$. In particular, for each index $k, h_{n}^{\prime}$ has an orbital contained in $B_{n, k}$ that shares an end with $B_{n, k}$, so that $h_{n}^{\prime}$ must have $B_{n, k}$ as an orbital by points 3 (a)-(c) of Lemma 2.7 .

Also observe from Lemma 2.7 that any orbital of $h_{n-1}$ which properly contains an orbital of $g_{n}$ will now be an orbital of $h_{n}^{\prime}$, and that any orbital of $g_{n}$ that properly contains an orbital of $h_{n-1}$ will also be an orbital of $h_{n}^{\prime}$. If $B$ is an orbital of $h_{n-1}$, which has trivial intersection with the orbitals of $g_{n}$, then $B$ will be an orbital of $h_{n}^{\prime}$ as well. In particular, we have shown that $h_{n}^{\prime}$ now has an orbital containing every orbital of $h_{n-1}$.

For each index $n>0$, choose a positive integer $s_{n}$ large enough so that every orbital $C$ of $h_{n}^{\prime s_{n}}$ which properly contains orbitals of $h_{n-1}$ actually contains all such orbitals in a single fundamental domain of $h_{n}^{\prime s_{n}}$ on $C$.

Finally, define $h_{n}=h_{n}^{\prime s_{n}}$. By construction, we now have that the sequence $\left(h_{i}\right)_{i \in \mathbb{N}}$ of signatures satisfies the following list of properties:

(1) For each $n \in \mathbb{N}, A_{n}$ is an orbital of $h_{n}$. 
(2) For each $n \in \mathbb{N}$ with $n>0$, the orbitals of $h_{n-1}$ in $A$ are all contained inside a single fundamental domain of $h_{n}$ in $A_{n}$.

(3) For each $n \in \mathbb{N}$ with $n>0$, if $B$ is an orbital of $h_{n}$ which is not disjoint from the orbitals of $h_{n-1}$, then there are two possibilities.

(a) $B$ is also an orbital of $h_{n-1}$.

(b) $B$ properly contains a non-empty collection of orbitals of $h_{n-1}$ in a single fundamental domain of $h_{n}$ on $B$.

In particular, we can form the a new exemplary tower $E_{3}=\left\{\left(A_{i}, h_{i}\right) \mid i \in \mathbb{N}\right\}$.

To recap, $E_{3}$ has the properties that $\bigcup_{i \in \mathbb{N}} A_{i}=A$ and $A$ is an orbital of the group $\left\langle S_{E_{3}}\right\rangle$. Further, the signatures satisfy the three enumerated points above.

Now create tower $E_{4}$, which is the result of applying the growing tower operation of Lemma 3.5 to $E_{3}$. (We will always assume re-indexing when passing to a growing sub-tower.) The tower $E_{4}$ now has the property that if $\left(B, h_{i}\right)$ is a non-terminal signed orbital with respect to $E_{4}$, then $B$ is properly contained in an orbital of the signature $h_{i+1}$ of $E_{4}$.

Stage 5: Removing terminal orbitals. The tower $E_{4}$ is far superior to the initial tower $E_{0}$, but $h_{0}$ may still have terminal orbitals.

Suppose that $h_{0}$ does have some terminal orbitals. Then there is $N_{0} \in \mathbb{N}$ so that all the terminal orbitals of $h_{0}$ are stable for $n \geq N_{0}$ (that is, if $B$ is a terminal orbital of $h_{0}$ then there is an orbital $C$ of $h_{N_{0}}$ with $B \subseteq C$ so that for all $n \geq N_{0}, h_{n}$ also has orbital $C$ ).

Compute a new element $k=\left[\left[h_{N_{0}+1}, h_{N_{0}+2}\right], h_{N_{0}+2}\right]$ (note that condition (3) above implies that $h_{N_{0}+1}$ and $h_{N_{0}+2}$ satisfy the mutual efficiency condition, since all the orbitals of $h_{j}$ are contained in orbitals of $h_{j+1}$ for any $j \in \mathbb{N}$ ). By Lemma 2.9, the element $k$ has the following properties:

(1) The orbitals of $h_{N_{0}}$ which contain the terminal orbitals of $h_{0}$ are not contained in the union of the orbitals of $k$.

(2) No orbital of $h_{N_{0}}$ which is also an orbital of $h_{N_{0}+1}$ is an orbital of $k$. (Note that these are all terminal orbitals of $h_{N_{0}}$ since $E_{4}$ is the result of using a growing tower operation).

(3) All the non-terminal orbitals of $h_{N_{0}}$ are still properly contained in the orbitals of $k$, since $k$ contains the non-terminal orbitals of $h_{N_{0}+1}$.

Now replace $k$ and $h_{N_{0}}$ by sufficiently high powers of themselves so that they satisfy the mutual efficiency condition. Define $h=\left[\left[h_{N_{0}}, k\right], k\right]$. By Lemma 2.9, the element $h$ has the following properties:

(1) $h$ has no orbitals intersecting the terminal orbitals of $h_{0}$.

(2) $h$ has all the non-terminal orbitals of $h_{N_{0}}$. 
Now replace $h$ and $h_{0}$ by sufficiently high powers of themselves so that the satisfy the mutual efficiency condition, and then replace $h_{0}$ by $\left[\left[h_{0}, h\right], h\right]$. By Lemma 2.9, every orbital of the new $h_{0}$ is properly contained in a non-terminal orbital of $h_{N_{0}}$, and every non-terminal orbital of the signature $h_{0}$ from the tower $E_{4}$ is also a non-terminal orbital of the new signature $h_{0}$.

Now replace $h_{N_{0}}$ by a sufficiently high power of itself so that $h_{0}$ and the new $h_{N_{0}}$ satisfy the mutual efficiency condition, and build the tower

$$
E_{5}^{\prime}=\left\{\left(A_{0}, h_{0}\right)\right\} \cup\left\{\left(A_{i}, h_{i}\right) \mid i \geq N_{0}, i \in \mathbb{N}\right\} .
$$

Create $E_{5}$ by re-indexing $E_{5}^{\prime}$ in the natural, order-preserving fashion. The tower $E_{5}$ satisfies all the properties of the tower $E_{4}$ (listed near the end of the previous stage), but its bottom element $\left(h_{0}\right)$ has only non-terminal orbitals. (Note that if the original $h_{0}$ had some terminal orbitals, then the new $h_{0}$ will have some new orbitals contained properly in the non-terminal orbitals of $h_{1}$.)

If the signature $h_{0}$ of $E_{4}$ had no terminal orbitals, then simply define $E_{5}$ to be $E_{4}$.

We can now repeat this whole process for the sub-tower of $E_{5}$ starting from index one, so that the new $h_{1}$ will admit all the non-terminal orbitals that it started with, and possibly other non-terminal orbitals, and also will contain no terminal orbitals. Further, replace $h_{1}$ by a high power of itself so that $h_{1}$ and $h_{0}$ are mutually efficient. Now inductively proceed up the tower $E_{5}$, redefining each of the $h_{i}$ in like fashion as we did for $h_{1}$ (using the tower consisting of signed orbitals with indices $k \geq i$ to find an $h_{i}$ with only non-terminal orbitals, and which has orbitals containing all of the orbitals of $h_{i-1}$, and then replacing the new $h_{i}$ with a high power of itself to guarantee mutual efficiency with $\left.h_{i-1}\right)$, to build a new tower $E_{6}=\left\{\left(A_{i}, h_{i}\right) \mid i \in \mathbb{N}\right\}$ which satisfies the following properties:

(1) $A=\bigcup_{n \in \mathbb{N}} A_{n}$.

(2) For each $n \in \mathbb{N}$ with $n>0$, the orbitals of $h_{n-1}$ in $A$ are all contained inside the orbital $A_{n}$ of $h_{n}$.

(3) For each $n \in \mathbb{N}$ with $n>0$, if $B$ is an orbital of $h_{n}$ which is not disjoint from the orbitals of $h_{n-1}$, then $B$ contains the closure of the union of the collection of orbitals of $h_{n-1}$ that intersect $B$.

(4) For every index $n$, the elements $h_{n}$ and $h_{n+1}$ are mutually efficient.

Stage 6: Notes on dynamics with algebraic conclusions. For every $n \in \mathbb{N}$, define the subgroup $H_{n}=\left\langle h_{0}, h_{1}, \ldots, h_{n}\right\rangle$ of $G$. Now suppose that $n>0$. Given any two elements $f, g \in H_{n-1}$, since the supports of $f$ and $g$ are contained in the support of $h_{n-1}$, and since the support of $h_{n-1}$ in any one orbital of $h_{n}$ is contained in a single fundamental domain of $h_{n}$ in that orbital, we see that $f^{h_{n}^{j}}$ and $g^{h_{n}^{k}}$ have disjoint supports and therefore commute, whenever $j \neq k$. Since $H_{n-1} \cong H_{n-1}^{h_{n}^{j}}$ for any integer $j$, we therefore see that the subgroup of $H_{n}$ consisting of finite products of 
conjugates of elements of $H_{n-1}$ by $h_{n}$ is isomorphic to $\bigoplus_{j \in \mathbb{Z}} H_{n-1}$. Now, as we can write any element of $H_{n}$ as a product of an integer power of $h_{n}$ with a product of conjugates of elements of $H_{n-1}$ by integer powers of $h_{n}$ (which each commute with each other when different powers of $h_{n}$ are involved in the conjugations), we see that $H_{n} \cong H_{n-1}\left\langle\mathbb{Z}\right.$, where the $\mathbb{Z}$ factor is the subgroup $\left\langle h_{n}\right\rangle$ of $H_{n}$.

Now $H_{0} \cong \mathbb{Z}$, so $H_{1} \cong \mathbb{Z}<\mathbb{Z}, H_{2} \cong(\mathbb{Z}<\mathbb{Z})<\mathbb{Z}$, and etc., so that $H_{n} \cong$ $(\cdots((\mathbb{Z} \backslash \mathbb{Z}) \succ \mathbb{Z}) \cdots>\mathbb{Z}$ where the finite wreath product has $n+1$ factors of $\mathbb{Z}$. In particular, the ascending union of the $H_{n}$ is $H=\left\langle h_{0}, h_{1}, \ldots\right\rangle \cong(\mathbb{Z} \imath)^{\infty}$.

Lemma 3.7. If $G$ is a subgroup of $\mathrm{PL}_{o}(I)$ and $G$ admits a deep tower, then $G$ has a subgroup of the form ${ }^{\infty}(2 \mathbb{Z})$.

Proof. We will use a similar technique to the proof of Lemma 3.6, although the analysis in this case is much simpler.

If $G$ admits a transition chain of length two, then by Theorem 1.4, $G$ admits an embedded copy of $B$, and $B$ contains copies of $\infty$ ( $2 \mathbb{Z})$. So let us assume that $G$ admits no transition chains of length two.

Since $G$ is admits no transition chains of length two, any tower for $G$ is exemplary. In particular, let $E=\left\{\left(A_{-i}, g_{-i}\right) \mid i \in \mathbb{N} \backslash 0\right\}$ be an exemplary deep tower for $G$ where the indexing respects the order on the elements of the tower. Improve $E$ by replacing the signatures of $E$ with sufficiently high powers of themselves so that given any negative integer $i$, then $g_{i-1}$ and $g_{i}$ satisfy the mutual efficiency condition.

Let $A=A_{-1}=(a, b)$. Since $E$ is exemplary, we see that $A$ is actually an orbital of the subgroup $H \leq G$, where $H=\left\langle S_{E}\right\rangle$. For all $i \in \mathbb{N}$ with $i>1$, inductively improve $E$ (induct on increasing $i \in \mathbb{N}$ in the following discussion) by replacing the signatures of $E$ according to the following three step process.

First, let $h_{-i}=\left[\left[g_{-i}, g_{-i+1}\right], g_{-i+1}\right]$.

Second, define the new $g_{-i}$ to be $h_{-i}$.

Third, replace the elements $g_{-i+1}, g_{-i}$ and $g_{-i-1}$ with sufficiently high powers of themselves, so that given any index $j \in \mathbb{N}$, the elements $g_{-j}$ and $g_{-j-1}$ satisfy the mutual efficiency condition. (Observe that if $i>3$, then $g_{-i+1}$ and $g_{-i+2}$ will now still satisfy the mutually efficiency condition, since we are only replacing $g_{-i+1}$ by higher powers of itself, and these two signatures were already mutually efficient; a similar argument shows that $g_{-i-1}$ and $g_{-i-2}$ will be mutually efficient after this operation as well.)

Since $A_{-i} \subsetneq A_{-i+1}$ for all integers $i>1$, we see that the resultant set of signed orbitals is still a tower (and with the same order), so that this inductive definition will simply improve our tower $E$. Observe further that given any $k \in \mathbb{N}$, then the orbitals of $g_{-k-1}$ are all properly contained in the orbitals of $g_{-k}$.

Define the set $\Gamma_{i}=\left\{g_{j} \mid j \leq i, j \in \mathbb{Z}\right\}$ for each negative integer $i$. For each negative integer $i$, define $H_{i}=\left\langle\Gamma_{i}\right\rangle$. For such $i$, the orbitals of $H_{i}$ are actually the orbitals of $h_{i}$, since all orbitals of the elements $g_{k}$ with $k<i$ are contained in the 
orbitals of $g_{i}$. Furthermore, for any such $i<-1$, the orbitals of $g_{i}$ are contained in the orbitals of $g_{i+1}$ in such a way that in any individual orbital $B$ of $g_{i+1}$, the support of $g_{i}$ in $B$ is actually fully contained inside a single fundamental domain of $g_{i+1}$ on $B$. In particular, $H_{i} \cong H_{i-1} \prec \mathbb{Z}$, where the $\mathbb{Z}$ factor comes from the subgroup $\left\langle g_{i}\right\rangle$ of $H_{i}$. But now inductively, since each generator generates a group isomorphic to $\mathbb{Z}$, we see that $H_{1} \cong \infty(2 \mathbb{Z})$.

Lemma 3.8. If $G$ is a subgroup of $\mathrm{PL}_{o}(I)$ and $G$ admits a bi-infinite tower, then $G$ has a subgroup of the form $(২ \mathbb{Z} 乙)^{\infty}$.

Proof. This follows immediately from the previous two lemmas, where first one improves the non-negative tower and then improves the negative tower (using the element with index 0 as the top element).

3.3. $W$ in arbitrary non-solvable subgroups of $\mathbf{P L}_{\boldsymbol{o}}(I)$. We are now ready to complete the proof of Theorem 1.1, restated below.

Theorem 1.1. Let $H$ be a subgroup of $\mathrm{PL}_{o}(I) . H$ is non-solvable if and only if $W$ embeds in $H$.

Proof. Since $W$ is non-solvable, any group which contains an embedded copy of $W$ will be non-solvable as well, therefore we need only show that if $H$ is non-solvable then $H$ contains a copy of $W$.

Suppose therefore that $H$ is a non-solvable subgroup of $\mathrm{PL}_{o}(I)$. By Lemma 3.1 we know that $W$ embeds in ${ }^{\infty}(\iota \mathbb{Z})$ and $(\mathbb{Z} \iota)^{\infty}$, and therefore also into $(\imath \mathbb{Z} \imath)^{\infty}$. Therefore, if $H$ admits infinite towers then we already have the result, so let us assume that $H$ does not admit infinite towers. In this case, by Theorem 1.5, we have that $H$ admits towers of arbitrary finite height. By the proof of Theorem 1.4, if $H$ admits a transition chain of length two, then it admits a bi-infinite tower (the tower is built in stage 8, p. 26). In particular, we may assume that $H$ admits no transition chains of length two. Now Lemma 2.7 guarantees us that $H$ is balanced.

Since $H$ does not admit infinite towers, the depth of any signed orbital of $H$ is well defined and finite. Since $H$ is not the trivial group, $H$ has a non-empty collection of orbitals. The analysis now breaks into two cases.

Case 1. $H$ admits no orbital that supports towers of arbitrary height. In this case we may define the depth of any orbital $B$ of $H$ as the maximum height of the towers which are supported by the orbital $B$. Since $H$ admits towers of arbitrary finite height, but no orbitals supporting towers of arbitrary height, we see that $H$ has must admit infinitely many orbitals. In particular, $H$ admits orbitals with depth greater than $n$ for any natural number $n$.

Now pick an element $\hat{g}_{1,1}$ of $H$ so that $\widehat{T}_{1}=\left\{\left(B_{1,1}, \hat{g}_{1,1}\right)\right\}$ is a tower of height one for $H$. The element $\hat{g}_{1,1}$ has finitely many orbitals, and so there is a maximum 
depth $j_{1}$ of the orbitals of $H$ that are not disjoint from the support of $\hat{g}_{1,1}$. We will now pick our remaining generators from the group $H^{\left(j_{1}\right)}$, the $j_{1}$ 'st derived subgroup of $H$. We note that no element in $H^{\left(j_{1}\right)}$ can have support intersecting $\hat{g}_{1,1}$, since $H^{\left(j_{1}\right)}$ has trivial support over the orbitals of $H$ of depth less than or equal to $j_{1}$ as a consequence of the details of the proof of point (4) of Lemma 2.7. (We state the main idea of that proof, which is in [3]. If $\iota$ is an element of $H$ and $(Z, \iota)$ is a depth one signed orbital of $H$, then there is no element of $H$ with an orbital containing $\bar{Z}$, the closure of $Z$. In particular, $Z$ cannot be the orbital of any commutator of elements of $H$, nor of a finite product of commutators. Hence, each time we pass to a commutator subgroup, we lose all elements which support the depth one orbitals of the original group.)

We also observe that $H^{\left(j_{1}\right)}$ still admits towers of arbitrary finite height and infinitely many orbitals of arbitrary finite depth. For $H^{\left(j_{1}\right)} \leq H$ we now find a tower $\widehat{T}_{2}=\left\{\left(B_{2,1}, \hat{g}_{2,1}\right),\left(B_{2,2}, \hat{g}_{2,2}\right)\right\}$ of height two. Now the signatures of $\widehat{T}_{2}$ admit a finite total number of orbitals, and therefore the union of this collection of element orbitals is contained in the union of the collection of orbitals of $H$ of depth less than some integer $j_{2}>j_{1}$. We therefore will pick a tower $\widehat{T}_{3}=$ $\left\{\left(B_{3,1}, \hat{g}_{3,1}\right),\left(B_{3,2}, \hat{g}_{3,2}\right),\left(B_{3,3}, \hat{g}_{3,3}\right)\right\}$ for $H^{\left(j_{2}\right)}$ which has signatures whose supports must be disjoint from the supports of the signatures of the first two towers $\widehat{T}_{1}$ and $\widehat{T}_{2}$ (by using elements from $H^{\left(j_{2}\right)}$, for example). We can continue in this fashion to inductively define towers $\widehat{T}_{k}$ and integers $j_{k-1}$ for each positive integer $k$ so that the integers $j_{k}$ are always getting larger, and so that the towers $\widehat{T}_{k}$ always have height $k$ and have signatures which are disjoint in support from the signatures of the previous towers. (We insist that in the construction above the indexing always respects the ordering of the signed orbitals in all of the towers we build here, so that $A_{k, i} \subset A_{k, i+i}$ for any index $k$ and $i$ with $1 \leq i<k$.)

Let $g_{1,1}=\hat{g}_{1,1}$. For each index $k>1$ replace the signature $\hat{g}_{k, k}$ and $\hat{g}_{k, k-1}$ of the tower $\widehat{T}_{k}$ by a sufficiently high powers of themselves so that pairwise, they are mutually efficient. Now build a new tower $T_{k}=\left\{\left(A_{k, 1}, g_{k, 1}\right),\left(A_{k, 2}, g_{k, 2}\right), \ldots,\left(A_{k, k}, g_{k, k}\right)\right\}$, where $g_{k, k}=\hat{g}_{k, k}$ and where we inductively define $g_{k, i}$ in the following fashion. Starting with index $i=k-1$, define $g_{k, i}=\left[\left[\hat{g}_{k, i}, g_{k, i+1}\right], g_{k, i+1}\right]$. Now replace $g_{k, i}$ and $\hat{g}_{k, i-1}$ by sufficiently high powers of themselves so that they are mutually efficient. Now inductively repeat for each smaller index $i$ the process just carried out for $i=k-1$ until $g_{k, 1}$ is defined (of course, we do not need to raise $g_{k, 1}$ to high powers, as there is no element $\hat{g}_{k, 0}$ ).

By construction, and by repeated application of Lemma 2.9, the process in the last paragraph results in elements $g_{k, j}$ so that if $k, i$ are indices with $1 \leq i<k$ then

(1) all orbitals of $g_{k, i}$ are properly contained in orbitals of $g_{k, i+1}$,

(2) $A_{k, i}$ is an orbital of $g_{k, i}$, and

(3) if $B$ is any orbital of $g_{k, j}$ for an index $1<j \leq k$, and $B$ contains an orbital 
of $g_{k, j-1}$, then all orbitals of $g_{k, j-1}$ in $B$ are contained in a single fundamental domain of $g_{k, j}$.

The points above together imply that for any index $k>0$, if we define $G_{k}=$ $\left\langle g_{k, 1}, g_{k, 2}, \ldots, g_{k, k}\right\rangle$, then $G_{k} \cong W_{k}$.

Now, as the support of every element of $G_{k}$ is disjoint from the support of each element of $G_{j}$ when $j \neq k$, we see that for such indices the union of the generators of the groups $G_{k}$ and $G_{j}$ will generate a group isomorphic with $G_{k} \oplus G_{j} \cong W_{k} \oplus W_{j}$. It is now immediate that the union of all of the generators of the groups $G_{j}$ will generate a group isomorphic with $W$.

Case 2. $H$ admits an orbital $A$ that supports towers of arbitrary height. If $A$ is not an orbital of any element of $H$ then $A$ can be written as a union of an infinite collection of nested element orbitals of $H$, so that $H$ would then admit an infinite tower, therefore there is an element $d$ of $H$ so that $(A, d)$ is a signed orbital of depth one for $H$.

We will now restrict our attention to a special subgroup $H_{d}$ of $H$ which is directed by the element $d$, in a sense that will be made clear. Given any element $h \in H$, let $k_{h}$ and $j_{h}$ represent the smallest positive integers so that $h^{k_{h}}$ and $d^{j_{h}}$ satisfy the mutual efficiency condition. Let

$$
\Gamma_{d}=\left\{\left[\left[h^{k_{h}}, d^{j_{h}}\right], d^{j_{h}}\right] \mid h \in H\right\} \cup\{d\} .
$$

The elements of $\Gamma_{d}$ have all of their orbitals properly contained inside the orbitals of $d$, and since the orbital $A$ of $H$ admits towers of arbitrary height and any element orbital $B$ which is properly contained inside $A$ will be realized as an orbital of some element $g$ of $\Gamma_{d}$ (note that it does not matter that we passed to high powers to guarantee the mutual efficiency condition), we see that the group $H_{d}=\left\langle\Gamma_{d}\right\rangle$ admits towers of arbitrary height.

Observe that given any finite set $X$ of elements of $H_{d}$ that individually do not support any signed orbitals of depth one for $H_{d}$, and a finite tower $T$ for $H_{d}$ which also contains no signed orbital of depth one, we can find a minimal power $k_{X}$ of $d$ so that the tower $T^{d^{k} X}$ for $H_{d}$ induced from $T$ via conjugation of the signatures of $T$ by $d^{k_{X}}$ will have all of its signatures having disjoint support from the signatures of $X$. (To do this, we choose $k_{X}$ to be large enough so that the supports of the signatures of $T^{d^{k}}$ are nearer to the ends of the orbitals of $d$ than the supports of the elements of $X$ ).

Now, for each positive integer $n$, let $\widetilde{T}_{n}$ be a tower for $H_{d}$ of height $n$. Now inductively define towers $\widehat{T}_{n}$ which are towers induced from the $\widetilde{T}_{n}$ by conjugation by powers of $d$ so that given any positive integer $k$, the tower $\hat{T}_{k}$ has signatures whose supports are all disjoint from the supports of the signatures of the towers $\widehat{T}_{j}$ for indices $j$ with $1 \leq j<k$. integer.

By treating the towers $\widehat{T}_{n}$ as the towers with the same names were treated in the discussion in Case 1, we can again find an embedded copy of the group $W$ in $H$. 


\section{References}

[1] C. Bleak, Solvability in groups of piecewise-linear homeomorphisms of the unit interval. Dissertation, Binghamton University, New York 2005. arXiv:math/0510399

[2] C. Bleak, An algebraic classification of some solvable groups of homeomorphisms. J. Algebra 319 (2008), 1368-1397. Zbl 05264500 MR 2383051

[3] C. Bleak, A geometric classification of some solvable groups of homeomorphisms. J. London Math. Soc. (2) 78 (2008), 352-372. Zbl 05359602 MR 2439629

[4] M. G. Brin, The ubiquity of Thompson's group $F$ in groups of piecewise linear homeomorphisms of the unit interval. J. London Math. Soc. (2) 60 (1999), 449-460. Zbl 0957.20025 MR 1724861

[5] M. G. Brin, Elementary amenable subgroups of R. Thompson's group F. Internat. J. Algebra Comput. 15 (2005), 619-642. Zbl 1110.20018 MR 2160570

[6] M. G. Brin and F. Guzmán, Automorphisms of generalized Thompson groups. J. Algebra 203 (1998), 285-348. Zbl 0930.20039 MR 1620674

[7] M. G. Brin and C. C. Squier, Groups of piecewise linear homeomorphisms of the real line. Invent. Math. 79 (1985), 485-498. Zbl 0563.57022 MR 782231

[8] M. G. Brin and C. C. Squier, Presentations, conjugacy, roots, and centralizers in groups of piecewise linear homeomorphisms of the real line. Comm. Algebra 29 (2001), 4557-4596. Zbl 0986.57025 MR 1855112

[9] K. S. Brown, Finiteness properties of groups. J. Pure Appl. Algebra 44 (1987), 45-75. Zbl 0613.20033 MR 0885095

[10] J. Burillo, S. Cleary, and M. I. Stein, Metrics and embeddings of generalizations of Thompson's group F. Trans. Amer. Math. Soc. 353 (2001), 1677-1689. Zbl 0989.20030 MR 1806724

[11] M. Stein, Groups of piecewise linear homeomorphisms. Trans. Amer. Math. Soc. 332 (1992), 477-514. Zbl 0798.20025 MR 1094555

Received August 7, 2006; revised March 27, 2008

C. Bleak, Department of Mathematics, University of Nebraska at Lincoln, Lincoln, NE 86588-0130, U.S.A.

E-mail: cbleak2@math.unl.edu 